A paraître dans la revue : Recherches en Didactique des Sciences et des Technologies.

\title{
Des débats numériques pour développer les compétences argumentatives des élèves sur des questions socio-scientifiques?
}

Gabriel Pallares, gabriel.pallares.math@gmail.com

Manuel Bächtold, manuel.bachtold@umontpellier.fr

Valerie Munier, valerie.munier@umontpellier.fr

Université de Montpellier, LIRDEF (UM-UPVM)

Former les élèves à participer à des débats sur des questions socio-scientifiques (QSS) de façon éclairée est devenu un enjeu éducatif majeur. À cet égard, les outils numériques offrent de nouvelles possibilités pour mener des débats en classe. Mais quels sont précisément leurs apports, en particulier pour aider les élèves à appréhender la complexité des QSS et à développer leurs compétences argumentatives sur ces questions? Dans cet article, nous présentons l'expérimentation d'une séquence d'enseignement menée dans dix classes de lycée. Cette séquence est centrée sur un débat numérique. Celle-ci intègre l'usage d'une plateforme numérique de débat dont l'une des spécificités est d'utiliser un texte, et non une question, comme point de départ du débat. Pour analyser la qualité argumentative et la structure des débats, nous avons élaboré une grille d'analyse basée sur un modèle de l'argumentation intégrant ses dimensions dialogale et monologale. Nos analyses montrent qu'un débat numérique basé sur un texte aide les élèves à saisir ou faire émerger des questions qui font sens pour eux. Celui-ci leur permet également d'explorer collectivement et en parallèle les différentes dimensions de la QSS. En revanche, nous n'observons pas d'évolution significative des compétences argumentatives des élèves. Pour conclure, nous discutons des pistes de développement de notre travail, à la fois au niveau du dispositif didactique et sur le plan théorique et méthodologique.

Mots-clés : argumentation, débat, science technologie et société

Train students to take part in debates on Socio-Scientific Issues (SSI) has become a major concern. In this respect, computer-mediated debates provide new learning opportunities to carry out debates in classrooms. But what is precisely their contribution to help students develop argumentative skills on SSIs and grasp the complexity of these issues? In this study, we present and analyze the implementation of a teaching sequence in ten high school classes. This sequence is centered on a computer-mediated debate of which the starting point is a text and not a question. To assess the quality of argumentation and the structure of the debates, we developed an analysis grid based on a model of argumentation taking into account its dialogical and monological dimensions. Our analysis shows that a text-based computer- mediated debate helps students grasp and give rise to questions which are meaningful for them. It also allows them to tackle collectively and simultaneously the various dimensions of the SSI. However, we do not observe significant improvements of students' argumentative skills. In conclusion we discuss future directions of our work, concerning the teaching sequence and at the theoretical and methodological levels.

Keywords : argumentation, debate, science technology and society

Le projet AREN est soutenu par l'état dans le cadre du volet e-FRAN du Programme d'Investissements d'Avenir, opéré par la Caisse des dépôts et consignations. Nous tenons également à remercier ici tous les enseignants ayant pris et prenant part au projet AREN ainsi que les partenaires du projet : le laboratoire LIRMM, l'académie de Montpellier, l'ESPÉ de Montpellier, les sociétés Cartodébat, Mezoa et Intactile Design ainsi que les associations Forum des débats et Controversciences. 
A paraître dans la revue : Recherches en Didactique des Sciences et des Technologies.

\section{Argumentation et numérique : de nouveaux enjeux}

\subsection{L'argumentation sur les questions socio-scientifiques}

De nombreuses questions aujourd'hui cruciales pour le devenir de notre société mettent en jeu les sciences et les technologies: transition énergétique, développement durable, intelligence artificielle, avancées biotechnologiques, etc. Préparer les élèves, futurs citoyens, à prendre part de façon éclairée aux débats sur ces questions dites " socio-scientifiques " (ci- après QSS) est devenu un enjeu éducatif majeur (domaine 3 du socle de compétences, MEN, 2015 ; Gaussel, 2016). Cela ne renvoie pas seulement à un apprentissage de connaissances scientifiques ciblées, mais aussi et surtout à l'acquisition de différentes compétences argumentatives. II s'agit de développer chez les élèves une posture plus critique et responsable sur les sciences et leurs liens avec la société (Lange \& Victor, 2006), comme le préconise maintenant la Commission européenne (CE, 2012) avec son action Responsible Research and Innovation (RRI). Dans cette perspective d'éducation à la citoyenneté (ou «citoyenneté critique », Knippels, Van Dam \& Van Harskamp, 2017), les compétences argumentatives sont donc un objectif d'apprentissage, en même temps qu'elles sont des moyens pour sensibiliser les élèves aux QSS. En effet, se confronter à des QSS implique pour les élèves de mobiliser leurs compétences argumentatives. Réciproquement, débattre de ces questions offre une occasion de développer ces compétences, essentielles dans la formation des futurs citoyens (Breton, 2000 ; Gaussel, 2016). Cette double fonction de l'argumentation en tant que moyen et objectif d'apprentissage résonne avec les deux approches de l'argumentation dans l'enseignement des sciences mises en lumière par différents auteurs (Erduran \& Jiménez- Aleixandre, 2008 ; Schwarz \& Baker, 2015), « argumenter pour apprendre » et « apprendre à argumenter ». L'argumentation représente ainsi d'une part un moyen pour apprendre et conceptualiser les contenus scientifiques (Zohar \& Nemet, 2002 ; Weisser, Masclet \& Rémigny, 2003 ; Lhoste, 2006 ; Orange, Lhoste \& Orange-Ravachol, 2008) et d'autre part un objet à apprendre (Driver, Newton \& Osborne, 2000 ; Erduran, Simon \& Osborne, 2004 ; Clark \& Sampson, 2008).

De par leurs caractéristiques, les QSS constituent des sujets de débat potentiellement riches (Simonneaux, 2007 ; Simonneaux \& Simonneaux, 2011). En effet, à l'instar des Questions socialement vives (QSV) ${ }^{1}$, telles que définies par Simonneaux (2003) et Morin et al. (2014), elles sont complexes : les débats sur les QSS voient se confronter des arguments issus de domaines de connaissances variés (physique, biologie, mais aussi économie ou sociologie) ainsi que des valeurs plus ou moins partagées (Sadler \& Zeidler, 2005 ; Sadler \& Fowler, 2006 ; JiménezAleixandre, Eixeras \& Agraso, 2006). La multiplicité des domaines susceptibles d'être mobilisés lors du traitement d'une QSS peut ainsi induire une grande variété d'arguments. Soulignons qu'il ne suffit pas de prendre conscience des domaines mobilisés pour appréhender la complexité de la

\footnotetext{
1 Nous choisissons dans cet article de parler de «Questions socio-scientifiques » (QSS) plutôt que de «Questions socialement vives »(QSV), pour les deux raisons suivantes : d'une part, les QSV peuvent inclure des questions qui ne renvoient pas forcément aux sciences (Simonneaux, 2007, p. 183), incluant notamment des questions " sociosociologiques " comme la mondialisation, l'immigration ou le chômage ; d'autre part, les QSV impliquent une triple vivacité, au niveau de la communauté scientifique, de la société et de l'école (Simonneaux \& Simonneaux, 2005), ce qui n'est pas systématiquement le cas dans les questions que nous étudions ici.
} 
A paraître dans la revue : Recherches en Didactique des Sciences et des Technologies.

question, mais de comprendre et de s'approprier les arguments issus de ces différents domaines. De plus, ces questions sont ouvertes : les débats sur des QSS ne sont pas animés par la recherche d'une vérité mais admettent en général une pluralité de réponses possibles (Habermas, 2001), du fait notamment de l'implication de différents acteurs, ayant chacun des intérêts et des valeurs qui leur sont propres. Les QSS se prêtent ainsi à l'expression et à la confrontation des points de vue variés des élèves. Enfin, ces questions sont marquées par l'incertitude des connaissances mises en jeu dans les débats, pouvant concerner l'évolution future de la société, de la planète ou des technologies (Morin et al., 2014 ; Morin, Simonneaux \& Tytler, 2017). Prendre conscience des incertitudes peut alors se traduire par une intégration de celles-ci dans les différents arguments mobilisés, notamment par des nuances selon le degré de certitude des connaissances en jeu.

Cependant si les QSS sont susceptibles de donner lieu à des débats riches, elles sont également difficiles à appréhender pour les élèves. Pour débattre de manière riche et pertinente sur des QSS, les élèves doivent en effet acquérir de multiples compétences de haut niveau: compétences cognitives, langagières et psycho-sociales. Ils doivent être capables d'émettre un jugement (si possible nuancé) sur la question débattue (Kuhn, Hemberger \& Khait, 2016, p. 17). II leur faut prendre conscience qu'une thèse ne va pas de soi (Golder \& Favart, 2003, p. 188) et doit être justifiée (Erduran, Simon \& Osborne, 2004 ; Kuhn, Hemberger \& Khait, 2016, p. 20). En outre, ils doivent être capables de justifier leur point de vue, c'est-à-dire d'articuler par un raisonnement une thèse à une ou plusieurs justifications (Sadler \& Zeidler, 2005 ; Garcia-Mila \& Andersen, 2007). Ces justifications peuvent notamment reposer sur des données qu'il convient d'identifier et de mobiliser de manière adéquate (Kelly \& Takao, 2002 ; Sandoval \& Millwood, 2005). Ces justifications doivent de plus être logiquement valides et éviter l'écueil des nombreux types de fallacies (Zeidler, Lederman \& Taylor, 1992 ; Zeidler, 1997 ; Walton, 2010). Les élèves doivent également savoir écouter et être capables de prendre en compte le point de vue d'autrui, ce qui nécessite de comprendre qu'il existe des alternatives à son propre point de vue et des justifications qui peuvent les soutenir (Kuhn, Hemberger \& Khait, 2016, p. 6 et 21). Pour argumenter, et en particulier pour débattre, c'est-à-dire argumenter en situation de dialogue, les élèves doivent être capables de réfuter les thèses auxquelles ils s'opposent (Osborne, Erduran \& Simon, 2004 ; Clark \& Sampson, 2008) ou au moins de les nuancer, notamment par une prise en compte d'un domaine de validité et de potentielles incertitudes (Morin, Simonneaux \& Tytler, 2017). S'agissant plus spécifiquement des débats sur les QSS, l'un des enjeux est aussi que les élèves sachent distinguer les différentes dimensions de la question traitée (Simonneaux \& Simonneaux, 2011 ; Morin, Simonneaux \& Tytler, 2017 ; Beaufort et al., 2015). En outre, ils doivent savoir gérer les potentiels conflits (Plantin, 2016), avoir un esprit d'équipe ou faire preuve de leadership (Reverdy, 2016, p. 16, 18 et 20). Même si ce leadership n'est pas forcément synonyme d'absence de collaboration (Albe, 2008, p. 84), il ne doit pas s'exercer au détriment des autres et impliquer le monopole de la prise de parole (Simonneaux \& Simonneaux, 2005).

Plusieurs études empiriques ont porté sur la manière de développer les compétences argumentatives des élèves et de favoriser leur appréhension de la complexité des QSS. Elles ont permis de dégager deux pistes : 
A paraître dans la revue : Recherches en Didactique des Sciences et des Technologies.

1. Comme nous l'avons indiqué plus haut, le développement des compétences argumentatives et l'appréhension de la complexité des QSS sont favorisés par la pratique du débat (Kuhn, Shaw \& Felton, 1997 ; Kuhn \& Udell, 2003 ; Zohar \& Nemet, 2002 ; Erduran, 2007 ; Simonneaux, 2007 ; Morin et al., 2014 ; Morin, Simonneaux \& Tytler, 2017 ; Kuhn, Hemberger \& Khait, 2016);

2. Ils peuvent également être favorisés par un travail réflexif, c'est-à-dire d'ordre méta- cognitif (Zohar \& Nemet, 2002 ; Felton, 2004 ; Garcia-Mila \& Andersen, 2007 ; Schwarz \& Baker, 2017).

Dans une perspective d'éducation à la citoyenneté, une question didactique se pose alors : comment organiser des débats sur des QSS et mettre en place un tel travail réflexif en classe ? Selon Schwarz et Baker (2017, p. 22), le dispositif mis en place joue un " rôle majeur " pour permettre le développement d'une argumentation de qualité. Se focalisant sur les dispositifs mettant les élèves en débat, ces auteurs pointent un ensemble de " facteurs " qui peuvent influencer la qualité de l'argumentation (Schwarz \& Baker, ibid., chap. 6). Nous en exposons ici quelques-uns. Tout d'abord, le degré de problématisation : selon le dispositif, les élèves auraient plus ou moins l'occasion de s'approprier la question débattue. Pour ces auteurs, cette problématisation peut être favorisée si la question est construite par les élèves plutôt qu'imposée par l'enseignant. Ensuite, le guidage et la posture de l'enseignant durant le débat : il peut se mettre en retrait ou à l'inverse intervenir directement dans le débat pour apporter lui-même des contenus ou demander des explicitations, des reformulations, etc. Enfin, les connaissances préalables des élèves joueraient un rôle important sur la qualité de leur argumentation au cours du débat : celle-ci sera meilleure s'ils débattent sur une question ou un sujet à propos duquel ils possèdent déjà un minimum de connaissances (Sadler \& Fowler, 2006).

Ces dernières années, un ensemble d'études a mis en avant les usages possibles du numérique pour penser de tels dispositifs de débat et pour jouer sur ces différents facteurs (Clark et al., 2007 ; Schwarz \& Baker, 2017).

\subsection{Rôle du numérique}

Les plateformes numériques de débat offrent en effet un nouveau champ de possibles pour les débats en classe, notamment en permettant de circonscrire partiellement les obstacles sociaux, ce qui favorise la participation d'un grand nombre d'élèves en offrant du temps et un accès enrichi à l'information. De plus, un outil numérique permet de garder la trace écrite des échanges, et ainsi à la fois de prendre conscience de l'argumentation déployée et de l'analyser dans le cadre d'un travail réflexif méta-cognitif. Dans notre projet de recherche (détaillé plus bas), nous expérimentons, évaluons et développons la plateforme de débat en ligne AREN. Cet outil dispose de fonctionnalités originales : d'une part, le débat des élèves prend sa source dans un texte support, toujours accessible (figure 1) ; d'autre part, les élèves doivent reformuler les passages du texte ou les interventions de leurs camarades avant de déployer leur argumentation. Pour argumenter, l'élève doit en effet surligner le passage du texte ou de l'intervention d'un de ses 
A paraître dans la revue : Recherches en Didactique des Sciences et des Technologies.

pairs qu'il veut explorer, puis le reformuler, se positionner (d'accord/pas d'accord/pas compris), et argumenter pour justifier son positionnement (figure 2). Chaque positionnement correspond à un code couleur2 dans le champ de débat présenté en figure 1. Ce mode de fonctionnement induit une structure d'argumentation en arborescence (figure 3). Dans le cadre de cet article, nous n'explorerons pas la fonctionnalité de reformulation.

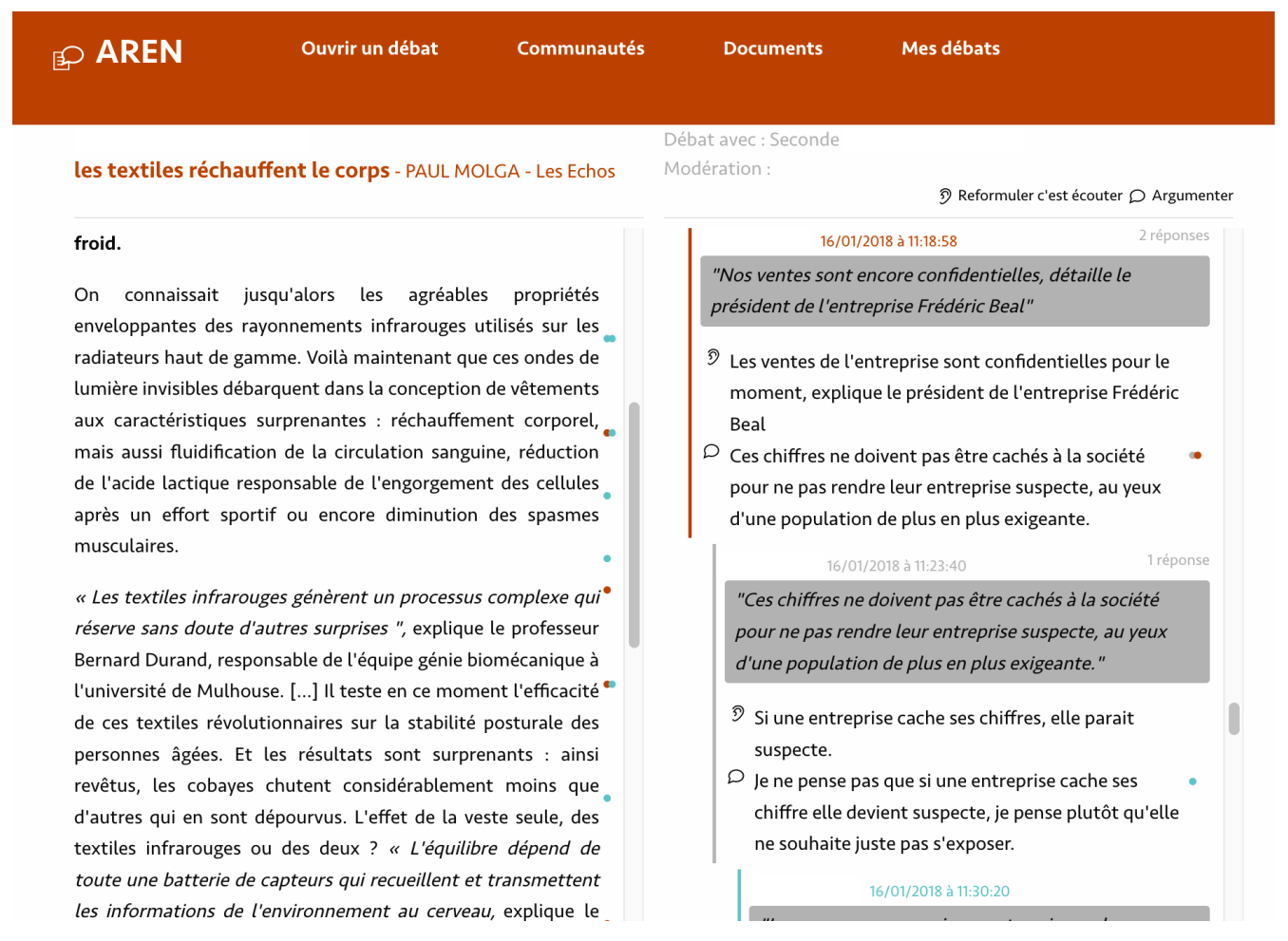

Fig. 1 : interface de débat de la plateforme AREN

2 Sur la plateforme, le positionnement «Pas d'accord » est codé en rouge, «D'accord » en bleu et «Pas compris » en gris. 
A paraître dans la revue : Recherches en Didactique des Sciences et des Technologies.

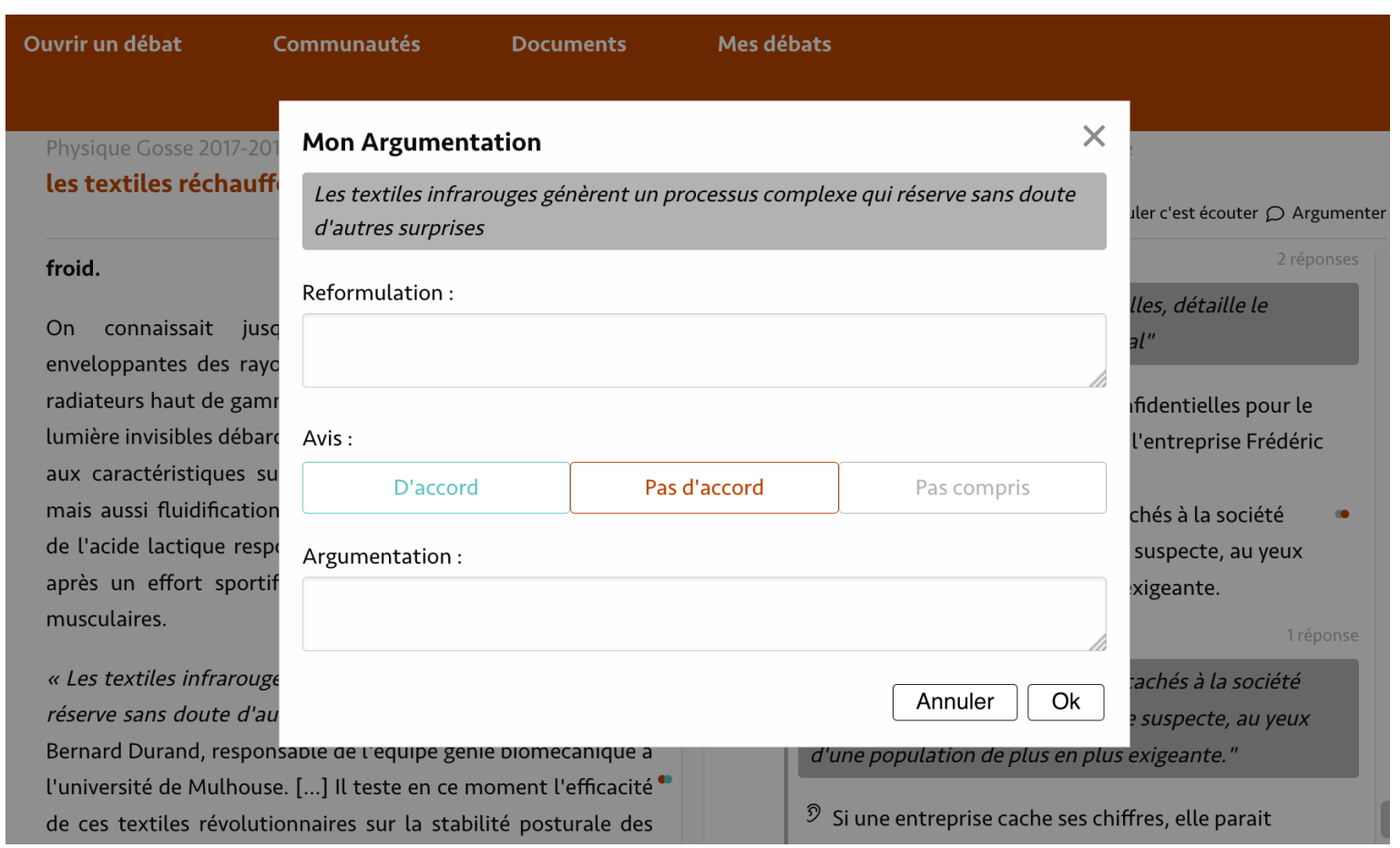

Fig. 2 : formulaire de reformulation et d'argumentation

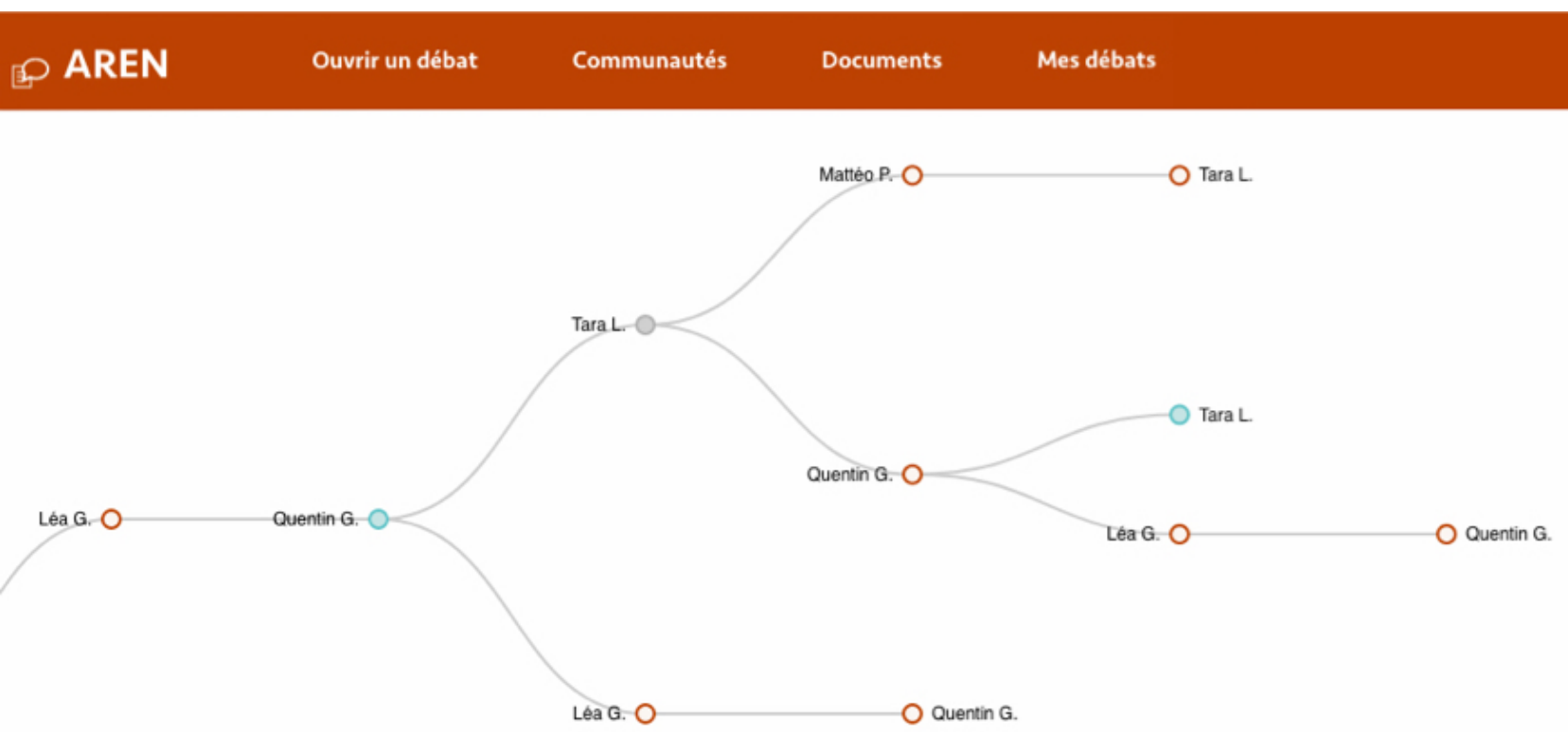

Fig. 3 : extrait d'une structure arborescente d'un débat

Notons que cet outil n'est pas figé : au contraire, l'un des objectifs du projet est d'améliorer et de développer la plateforme numérique en fonction des résultats de nos recherches. Par exemple, l'interface de la plateforme est en cours de modification pour permettre une plus grande lisibilité des échanges.

En appui sur la littérature déjà évoquée plus haut (notamment Schwarz \& Baker, 2017), nous considérons que la simple utilisation d'un outil numérique de débat n'est pas suffisante pour favoriser le développement des compétences argumentatives des élèves. Celui-ci doit être 
A paraître dans la revue : Recherches en Didactique des Sciences et des Technologies.

incorporé dans un dispositif didactique plus large, qui comprend d'une part une phase préalable permettant de préparer les élèves en termes de niveau des contenus pour pouvoir débattre et d'autre part une phase de synthèse leur permettant de mener un travail réflexif sur leurs propres productions durant le débat. Les outils numériques offrent ainsi de nouvelles possibilités pour mener des débats en classe. Mais quels sont plus précisément leurs apports ? Qu'en est-il en particulier dans le contexte éducatif précisé ci-dessus dans lequel il s'agit de sensibiliser les élèves aux QSS et de développer leurs capacités argumentatives ? Ces questions, qui font l'objet de la recherche présentée dans cet article, peuvent être formulées plus précisément de la manière suivante :

Quels sont les apports possibles d'un dispositif centré sur un débat numérique (faisant usage ici de la plateforme AREN) :

- pour appréhender la complexité des QSS ?

- pour aider les élèves à développer leurs compétences argumentatives sur des QSS ?

\section{2. Éléments théoriques}

La littérature portant sur l'argumentation est abondante, et relève de champs divers. L'argumentation est en effet un objet complexe pouvant être abordé selon différentes approches, lesquelles conduisent à des définitions multiples (Plantin, 2016).

\subsection{Sur l'argumentation}

Un renouveau des études sur l'argumentation a été initié durant la seconde moitié du XXe siècle notamment par Toulmin (1958), qui constitue une référence majeure pour les études sur l'enseignement de l'argumentation en éducation (Erduran, Ozdem \& Park, 2015). Cet auteur a proposé un modèle qui distingue les multiples composants possibles d'un argument. Celui-ci offre un moyen d'étudier et d'évaluer la structure d'un argument. Les travaux de Toulmin, en particulier son modèle d'un argument (en anglais TAP pour Toulmin's Argument Pattern) ont été beaucoup utilisés dans la littérature (Osborne et al., 2001 ; Erduran, Simon \& Osborne, 2004 ; Erduran, 2018). Précisons ici que le modèle de Toulmin décompose un argument en une donnée (factuelle), qui vient appuyer une thèse (défendue), par l'intermédiaire d'une garantie (représentant les liens entre les données et la thèse), elle-même soutenue par des fondements (loi générale venant soutenir la garantie). Certains arguments plus élaborés peuvent en outre contenir des restrictions présentant les conditions de validité et des modalisateurs venant détailler en quelle mesure la conclusion est certaine ou non au vu des données.

L'approche de Toulmin a depuis été critiquée (Simonneaux, 2003) et son modèle affiné (Erduran, Simon \& Osborne, 2004 ; von Aufschnaiter et al., 2008). Ces critiques renvoient à plusieurs caractéristiques essentielles de l'argumentation que nous discutons ci-dessous.

Tout d'abord, l'approche de Toulmin se focalise principalement sur l'argumentation en tant que produit, c'est-à-dire qu'il étudie les arguments individuels qui constituent ce qu'on pourrait appeler en français (le mot n'existant pas en langue anglaise) l'« argumentaire ». L'argumentaire, produit, 
A paraître dans la revue : Recherches en Didactique des Sciences et des Technologies.

est à distinguer de l'argumentation vue comme un processus (Greco- Morasso, Miserez-Caperos \& Perret-Clermont, 2015 ; Macagno, 2015 ; Henderson et al., 2018).

De plus, l'approche de Toulmin semble pertinente avant tout pour modéliser un argument construit par un seul individu, c'est-à-dire dans un contexte monologal, alors que de nombreux auteurs se penchent sur l'argumentation en contexte dialogal, construite en particulier au sein d'un débat (Jiménez-Aleixandre \& Erduran, 2007 ; Garcia-Mila \& Andersen, 2007 ; Polo, 2014 ; Plantin, 2016). Plusieurs auteurs insistent ainsi sur la spécificité des argumentaires produits en situation dialogale, notamment sur le rôle de la réfutation au sein des débats (Osborne, Erduran \& Simon , 2004 ; Clark \& Samson, 2008). Enfin, en négligeant l'aspect dialogal de l'argumentation, l'approche de Toulmin occulte un aspect important de l'argumentation, à savoir son but. Des auteurs comme Walton (1998) ont ainsi distingué notamment l'argumentation de type persuasif, compétitive et qui vise à emporter l'adhésion de son interlocuteur, de l'argumentation de type délibératif, coopérative et ayant plutôt pour but d'explorer une question. Le but attribué par les élèves au débat peut avoir des effets sur le type d'argumentation développé.

Cependant, d'une part, les recherches ne sont pas unanimes sur le type de débat le plus pertinent pour développer les compétences argumentatives (Schwarz \& Baker, 2017, p. 187- 188). D'autre part, nous considérons que cette distinction n'est pas aussi nette et qu'un débat globalement de type délibératif peut comporter des moments persuasifs et inversement : " polémicité et coopération caractérisent des moments du débat plus que des formes du débat en général » (Plantin, 2016).

Dans notre travail, nous nous positionnons dans la lignée de ces différentes critiques de Toulmin, en abordant l'argumentation sous l'angle à la fois d'un produit et d'un processus, selon ses aspects monologal (production individuelle d'arguments) et dialogal (interactions au sein d'un débat).

\subsection{Proposition d'une modélisation}

Notre exploration de la littérature nous a amenés à faire un certain nombre de choix théoriques qui nous permettent de proposer un modèle de l'argumentation dans le cadre d'un débat (figure 4). Ce modèle ne se veut ni définitif ni exhaustif, mais plutôt opératoire pour l'exploration des questions de recherche qui sont les nôtres. Pour des raisons de lisibilité, nous ne présentons pas le modèle dans le cadre d'un débat en classe entière mais nous l'explicitons dans le cas de deux interlocuteurs. 
A paraître dans la revue : Recherches en Didactique des Sciences et des Technologies.

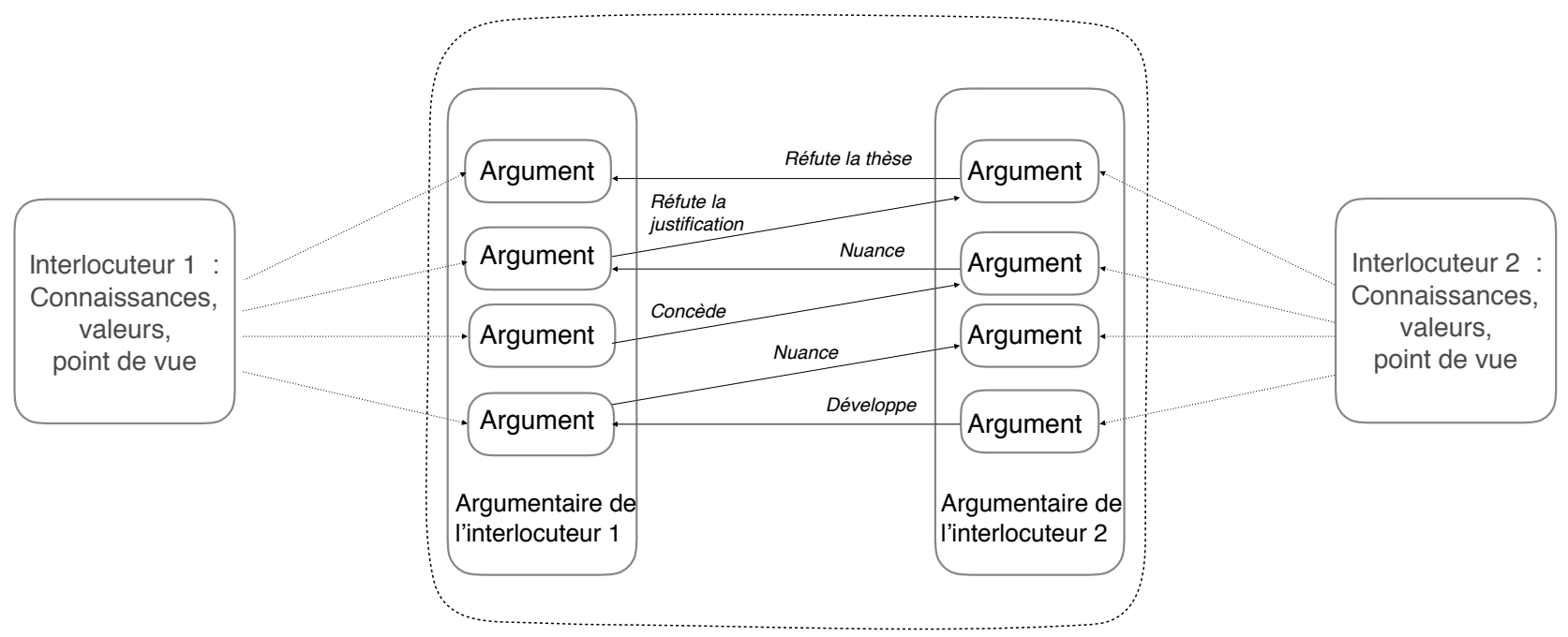

Fig. 4 : modélisation de l'argumentation au cours d'un débat entre deux interlocuteurs

Nous associons aux interlocuteurs d'un débat (ici, les élèves) des connaissances et des valeurs (qui leur permettent de proposer des justifications à leurs arguments) et un point de vue (qui représente la thèse et les éventuelles sous-thèses défendues lors du débat). Le processus argumentatif comporte deux dimensions : une dimension monologale, l'élève articulant ses connaissances propres avec son point de vue pour produire des justifications et ce faisant des arguments ; une dimension dialogale, l'élève produisant des arguments dans l'interaction, en fonction de l'avancée du débat et des notions déjà abordées. Nous avons ainsi distingué dans la figure 4 les argumentaires des deux interlocuteurs (le produit) et les interactions entre les arguments, qui renvoient à l'aspect dialogal du processus argumentatif. Ces interactions, annotées selon différentes modalités, représentent différentes possibilités d'interactions dialogales qui peuvent affecter l'argument : par exemple un argument visant à concéder et nuancer sera différent d'une réfutation formelle. Le but du débat est absent de cette représentation, la fonction de persuasion ou de délibération étant selon nous, comme nous l'avons dit précédemment, variable selon la consigne proposée par l'enseignant en début de séance, selon la perception que les élèves en ont, mais aussi selon le moment du débat.

Ce modèle présente l'argumentation comme un processus interactif qui permet aux interlocuteurs d'avancer collectivement dans l'exploration d'une question et la construction des points de vue ou des connaissances, voire de coopérer et/ou chercher un consensus (Reverdy, 2016). L'exploration conjointe d'une question ou la résolution d'un problème commun est aussi à prendre en compte pour envisager l'argumentation en classe, et les points de vue des élèves argumentant ne sont pas nécessairement fondamentalement antagonistes. À ce titre, notre modèle s'écarte de celui de Plantin (2016, p.527), appelé «Trilogue argumentatif », lequel suit une logique d'opposition et présente les actants de l'argumentation comme un triptyque «Proposant-Opposant- 
A paraître dans la revue : Recherches en Didactique des Sciences et des Technologies.

Tiers ${ }^{3}$. Ce modèle présente selon nous des limites pour évaluer les compétences argumentatives des élèves : comme Brossais, Panissal et Garcia-Debanc (2013, p. 131) l'ont souligné, une méthode d'analyse de l'argumentation à l'aune de ces trois rôles donne un statut ad hoc aux concessions et aux nuances dans la mesure où celles-ci ne peuvent pas être comprises comme éléments strictement propositionnels ou oppositionnels. Or nous considérons que les concessions et nuances méritent d'être relevées et valorisées en tant qu'indicateurs de la qualité argumentative. En outre, dans le Trilogue argumentatif de Plantin, la construction de la question est à la charge d'un "tiers ", alors que dans un contexte de classe la question peut être coconstruite tacitement par les élèves au fil du processus argumentatif (Baker, 2009b).

Rappelons ici que le modèle que nous proposons se veut heuristique, c'est-à-dire opératoire pour explorer des questions de recherche qui portent sur le développement de compétences argumentatives sur des QSS. Ce modèle est ainsi sous-tendu par une approche de l'argumentation comme objet propre à apprendre, plutôt que comme un moyen pour apprendre et construire des concepts nouveaux. II se focalise davantage sur le processus et la structure de l'argumentation que sur les contenus appris ou construits dans le cadre de l'argumentation. Pour étudier de façon détaillée l'élaboration collaborative des contenus par les élèves dans l'argumentation, d'autres modèles sont sans doute plus pertinents, par exemple celui des « macrostructures argumentatives » proposé par Orange, Lhoste et Orange-Ravachol (2008), lequel se base quant à lui sur les travaux de Plantin.

\section{Méthodologie}

Nous nous sommes appuyés sur les différents éléments théoriques présentés ci-dessus et en particulier sur notre modélisation de l'argumentation pour construire le dispositif didactique et la grille d'analyse de la qualité de l'argumentation. Dans cette partie, nous présentons ce dispositif, la démarche méthodologique employée et la grille d'analyse élaborée.

\subsection{Dispositif didactique}

Pour l'élaboration du dispositif didactique, nous avons suivi une méthodologie de Design Experiment (Cobb et al., 2003 ; Sandoval, 2013). Cette méthodologie implique une collaboration entre chercheurs et enseignants pour construire un dispositif qui prenne en compte à la fois les apports et questionnements de la recherche et les connaissances des enseignants à propos de la spécificité de l'argumentation au sein de leur discipline (en particulier sur l'usage des données), de la classe, des contraintes temporelles et organisationnelles de leur établissement, etc.

En outre, l'élaboration du dispositif est itérative : chaque année les retours d'expérience (tant les analyses des données que les retours des enseignants) vont contribuer à l'amélioration et l'affinement du dispositif de l'année suivante. Nous avons ainsi élaboré un dispositif comprenant trois phases :

\footnotetext{
3 II est à noter que Plantin ne considère pas le Proposant, l'Opposant et le Tiers comme des personnes nécessairement distinctes mais comme des rôles, qui peuvent être simultanément pris en charge par un même élève ou par des groupes d'élèves.
} 
A paraître dans la revue : Recherches en Didactique des Sciences et des Technologies.

- une phase de préparation des élèves au débat, visant à alimenter les élèves en termes de contenu ;

- le débat en classe à l'aide de la plateforme AREN ;

- une phase de synthèse du débat, permettant un bilan et un retour réflexif des élèves sur la qualité de leur argumentation dans le débat.

La phase de préparation au débat correspond à une étude de documents de différents types :analyse de vidéos (par exemple issues d'internet), de coupures de presse, d'articles de médiation scientifique, etc. Ces documents ont été choisis en collaboration avec les enseignants. Cette phase s'est déroulée sur 2 à 5 séances d'une heure de cours. Ce temps a été variable selon les disciplines et a principalement été fonction du temps alloué aux différents chapitres dans les programmes respectifs des disciplines (voir ci-dessous). Cette phase a été l'occasion pour les enseignants d'intégrer le dispositif didactique co-construit dans leur progression d'enseignement, en leur permettant d'aborder avec les élèves les contenus tout en les orientant plus spécifiquement sur le débat à venir.

Les phases de débat ont duré entre une heure et une heure et demie selon les possibilités des enseignants. Une fois fournies l'explication par les enseignants du fonctionnement de la plateforme, la résolution d'éventuels problèmes techniques et la passation de la consigne par les enseignants, les élèves ont effectivement débattu entre 20 et 60 minutes. Nous avons laissé aux enseignants le choix de la formulation de la consigne. Notre seule exigence a été de leur demander une consigne axée sur la délibération plutôt que sur la persuasion, avec comme visée la co-construction d'une compréhension commune du texte étudié et du thème abordé. Mis à part la passation de la consigne, nous avons demandé aux enseignants de se tenir plutôt en retrait durant la phase de débat et de limiter leurs interventions au maintien du bon déroulement du débat numérique. Toutefois, ils ont répondu aux questions posées par les élèves, notamment celles liées à la compréhension du texte débattu.

Lors de la phase de synthèse, les élèves ont analysé certaines interventions du débat (préalablement sélectionnées et imprimées par les enseignants), pour étudier les différents domaines de la QSS et la qualité de l'argumentation. Ce travail a été effectué par groupes de quatre élèves ; les groupes devaient s'accorder sur un classement des arguments selon leur justification (justifié ou non) puis devaient identifier les domaines dont relevaient les arguments justifiés. Pour aider les élèves, des exemples de domaines de la QSS étaient fournis par les enseignants, ces derniers ayant tous insisté au moins sur les aspects scientifiques, sociaux et économiques. Les groupes d'élèves devaient ainsi s'accorder au cours de leur discussion sur des critères permettant de classer les arguments. Quelques enseignants ont également fait élaborer aux groupes d'élèves un poster matérialisant leur classement dans un tableau. Ces critères étaient ensuite rediscutés en classe entière à l'occasion d'une mise en commun des travaux. Pour les enseignants qui n'avaient pas demandé de poster, cette mise en commun a pris la forme d'un tableau commun projeté à la classe, permettant d'organiser les arguments (ou des reformulations de ceux-ci). Précisons que, lors de la phase de synthèse, nous n'avons pas demandé aux enseignants une institutionnalisation sur l'argumentation en tant que telle. Nous avons privilégié 
A paraître dans la revue : Recherches en Didactique des Sciences et des Technologies.

une réflexion sur l'argumentation produite et sur la complexité des questions socio-scientifiques.

En effet, notre but était de stimuler la réflexion des élèves sur les arguments déployés lors du débat, sans nécessairement faire passer des points stabilisés à institutionnaliser. Cette institutionnalisation est de plus problématique dans le cas des QSS. En effet, elles sont complexes et mettent en jeu des savoirs de différents ordres et des valeurs pas toujours partagées. Elles sont de plus ouvertes et admettent plusieurs réponses possibles. Enfin, des incertitudes sont associées à ces questions, liées au fait que les savoirs en jeu ne sont pas tous stabilisés. Ces caractéristiques empêchent à notre sens une institutionnalisation, si ce n'est sur leurs caractères d'ouverture, d'incertitude et de complexité eux-mêmes.

\subsection{Participants}

Ce dispositif a été mis en œuvre dans 10 classes de lycée de l'académie de Montpellier, de niveaux variés à la fois en termes de niveau de classe (de la seconde à la terminale) et de milieu socio-culturel.

Les débats ont été mis en place au cours de l'année scolaire2016-2017, dans diverses disciplines et sur des thèmes différents :

- en géographie : débat sur les gaz de schistes (deux classes de seconde générale);

- en SVT : débat sur les liens entre action humaine et biodiversité (deux classes de seconde générale) ;

- en physique-chimie : débat sur la voiture électrique (trois classes de première scientifique) ;

- en éducation morale et civique (EMC) dans le cadre du cours de philosophie : débat sur la sélection des embryons (trois classes de terminale technologique).

Un rapide descriptif des questions abordées et des textes supports est proposé en annexes

1 et 2. La plupart des débats étaient organisés en demi-groupes : en tout 16 débats ont été organisés, impliquant un total de 259 élèves.

Le public enseignant était constitué de 6 femmes ( 1 enseignante récemment titularisée et 5 enseignantes expérimentées) et 4 hommes (tous expérimentés). Au vu de nos questions de recherche, l'objectif ici n'est pas de comparer les différents classes ou enseignants mais d'explorer les usages possibles de la plateforme AREN et leurs effets sur le développement des compétences argumentatives des élèves.

Nous avons fait le choix d'étudier des débats portant sur différentes QSS dans des disciplines diverses, non uniquement scientifiques. Ces questions diffèrent certes par leur échelle, leurs enjeux politiques, économiques et sociaux, les acteurs impliqués ou les objets de savoir mobilisés et leur épistémologie. Cependant, nous nous intéressons ici à ce qu'elles ont de commun, c'est-à-dire des caractéristiques d'ouverture, de complexité et d'incertitude, qui sont constitutives des QSS. Par ailleurs, un débat sur une QSS, quelle que soit la discipline, permet d'aborder des notions épistémologiques comme l'incertitude des connaissances, ou plus généralement de travailler avec les élèves leur vision des sciences. 
A paraître dans la revue : Recherches en Didactique des Sciences et des Technologies.

\subsection{Recueil de données}

Pour cette étude, nous avons analysé un débat par enseignant, soit 10 débats au total, impliquant un total de 167 élèves qui ont produit 1104 interventions. Parmi les débats analysés, 4 ont eu lieu en classe entière et 6 en demi-groupes. Après avoir filtré les interventions de type « bavardage $\aleph^{4}, 953$ ont été retenues pour l'analyse. Si nous ne nions pas leur utilité dans le débat, notamment pour permettre aux élèves d'alléger la charge cognitive pendant un débat d'une heure ou plus, il n'était pas pertinent de les coder avec une grille d'analyse applicable à l'argumentation. Nous avons également réalisé des pré- et post-tests, dans lesquels les élèves devaient se positionner sur une échelle de Likert par rapport à une assertion donnée, en lien avec le sujet du débat. Par exemple, en SVT, la phrase sur laquelle les élèves devaient se positionner était « II ne faut pas changer les activités humaines qui permettent le développement économique et social uniquement parce qu'elles pourraient faire disparaître des espèces animales ou végétales ». Les élèves devaient ensuite justifier leur position par rapport à la phrase dans un court paragraphe argumenté. Le fait de demander aux élèves de développer leur argumentation à partir d'une telle phrase visait à les rapprocher le plus possible d'une situation d'argumentation dialogale, qui correspond à celle de la séquence. Relativement au modèle présenté plus haut, la phrase donnée aux élèves a le statut d'une affirmation produite par un interlocuteur virtuel. Remarquons néanmoins que la situation du test diffère de celle du débat notamment dans la mesure où elle ne se caractérise pas par une dynamique d'échanges et où les dimensions sociales ou affectives sont atténuées, l'interlocuteur n'étant que théorique. Les élèves devaient également préciser sur une échelle s'ils comprenaient qu'on puisse avoir un avis différent. Pour le post-test, les élèves devaient en plus répondre à des questions visant à savoir s'ils pensaient avoir changé d'avis entre le prétest et le post-test, et pour quelles raisons ? Nous ne présentons pas les résultats des analyses de ces questions supplémentaires ici. Ces pré- et post-tests ont été administrés aux élèves par les enseignants. Notons que les conditions de passation n'ont pas toujours été celles prévues : les post-tests ont le plus souvent été réalisés à la fin de la séance de synthèse, sur un temps relativement réduit.

\subsection{Grille d'analyse micro des données}

Afin de mieux comprendre le processus d'argumentation à l'œuvre lors des débats numériques et des tests, nous analysons les arguments (pour les débats) et argumentaires (pour les tests) produits. La grille d'analyse repose sur la modélisation du débat présentée dans la figure 4 et les éléments de la littérature qui ont mené à l'élaboration de cette modélisation. En particulier, nous nous appuyons sur la distinction faite entre dimensions dialogale et monologale de l'argumentation afin de distinguer des items relevant plutôt de l'une ou de l'autre. Nous tentons ainsi, par une étude du " produit » " argument », d'évaluer la qualité du processus ayant participé à sa construction, l'argumentation.

\footnotetext{
${ }^{4}$ Nous avons choisi d'évacuer ces interventions car elles sont de fait à visée non argumentative.
} 
A paraître dans la revue : Recherches en Didactique des Sciences et des Technologies.

3.4.1. Indicateurs de la qualité argumentative

Au niveau des aspects dialogaux et interactionnels de l'argumentation lors d'un débat, nous évaluons dans les arguments la présence ou l'absence de réfutations de la thèse ou de la justification, vues comme spécifiquement orientées vers autrui. Notons qu'au sens que nous adoptons ici, la simple défense d'une thèse incompatible ou opposée n'est pas à proprement parler une réfutation. À la suite des travaux de Clark et Sampson (2008), nous avons choisi de distinguer l'opération de réfutation de la thèse d'autrui de celle de réfutation de la (ou des) justification(s) qui appuient cette thèse. En effet, il peut suffire dans une réfutation de la thèse de prendre en compte le point de vue d'autrui de façon globale et schématique. À l'inverse, dans une réfutation de la justification, l'élève doit appréhender précisément les arguments qui soutiennent la thèse d'autrui, du moins suffisamment pour pouvoir les évaluer et les réfuter. De plus, il est possible de réfuter les justifications avancées sans nécessairement être en opposition avec la thèse défendue : l'argument 2 présenté dans la figure 5 est une réfutation de la justification contenue dans l'argument 1 (" les hommes détruisent certains environnements pour construire des habitations »), sans qu'il n'y ait de réfutation de la thèse (" les hommes sont la cause de la disparition de la biodiversité »).

Nous étudions aussi la présence ou l'absence de nuances, vues comme des réfutations partielles ou "locales » de ce que propose autrui, sur des aspects périphériques ou un domaine d'application par exemple. Une nuance n'infirme pas totalement la parole de l'autre mais vient plutôt la moduler par un apport supplémentaire. Par exemple, l'argument 2 vient moduler l'argument 1 après l'avoir réfuté, en ajoutant l'idée de causes allant au-delà de la simple construction d'habitations.

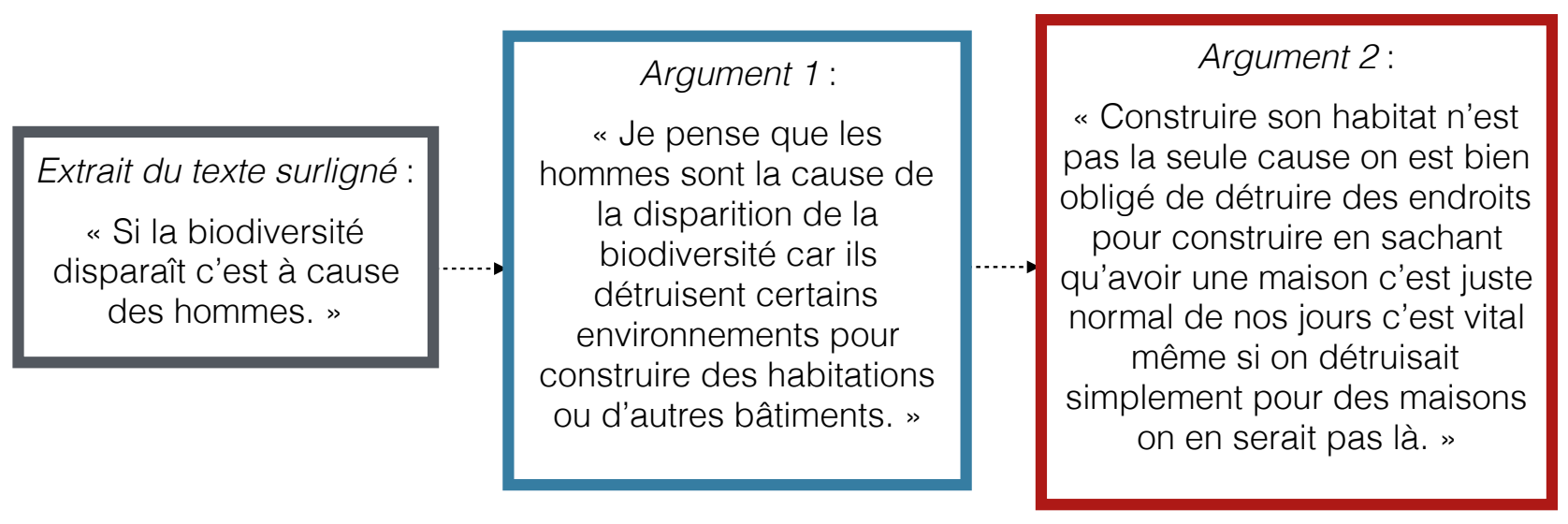

Fig. 5 : exemple d'une succession d'arguments extraite de notre corpus (débat en SVT en classe de seconde)

Nous codons également la présence ou l'absence de concessions, vues comme l'acceptation par un élève d'un élément controversé du discours d'autrui : thèse ou sous-thèse, nuance ou réfutation. Ainsi, dans la figure 5 , « construire son habitat n'est pas la seule cause on est bien obligé de détruire des endroits pour construire » suppose une concession : construire des 
A paraître dans la revue : Recherches en Didactique des Sciences et des Technologies.

habitations est au moins une des causes de destruction de l'environnement et donc de disparition de la biodiversité. L'argument 2 contient donc une concession, à l'inverse de l'argument 1 (qui ne pouvait en contenir puisqu'aucun élément de l'extrait du texte n'apparaît comme controversé).

Enfin, nous évaluons la présence dans les arguments des élèves de questionnements, c'est-àdire les questions posées par les élèves à leurs camarades lors du débat. Nous n'avons pas intégré dans cette catégorie les demandes de compréhension (notamment concernant des mots et expressions employées dans le texte) mais nous nous sommes principalement focalisés sur les questionnements critiques, les demandes d'explications et explicitations, ainsi que sur l'évocation de possibilités (" mais peut-être que... »).

En ce qui concerne la dimension monologale, qui se rapporte au raisonnement propre de l'élève lorsqu'il argumente lors d'un débat, nous avons évalué la présence de justifications et leur nombre. Un argument sera ainsi justifié s'il présente au moins une raison venant appuyer la thèse défendue dans l'argument. Les marqueurs langagiers " car », " donc », " parce que » peuvent aider au repérage des justifications, même s'ils ne sont pas suffisants dans la plupart des cas. Par exemple, si l'unique raison de l'argument 1 (figure 5) est facilement visible (« car ils détruisent... »), l'argument 2 contient 4 raisons réfutant la justification de son camarade sans connecteurs ni ponctuation : " on est bien obligé de détruire des endroits pour construire ", " en sachant qu'avoir une maison c'est juste normal de nos jours ", " c'est vital », " si on détruisait simplement pour des maisons on en serait pas là ".

Nous avons distingué les arguments justifiés des arguments non justifiés puis nous avons indiqué, pour les premiers, le nombre de raisons justifiant l'argument en regroupant les arguments présentant plus de trois raisons (les arguments présentant quatre raisons ou plus étant très peu nombreux).

Notons que nous n'avons pas pris en compte la pertinence des raisons invoquées par les élèves pour justifier leurs arguments. Si l'analyse de la pertinence est envisageable dans l'analyse de débats techno-scientifiques (Macagno, 2015), elle pose en revanche problème dans le cas de débats sur des QSS, où il est plus difficile de rapporter un argument à un savoir de référence, celui-ci étant complexe et marqué par des incertitudes, impliquant une grande part d'interprétation du chercheur. Nous avons donc choisi de cibler nos analyses sur la présence ou non de justifications (ou de tentatives de justifications) dans les interventions des élèves, considérant qu'il s'agit d'un critère important pour évaluer la qualité d'une argumentation. Nous avons en outre adapté et fusionné les restrictions et les modalisateurs de Toulmin (1958) en une catégorie visant à évaluer dans quelle mesure les élèves assignent ou non un domaine de validité à leurs assertions (leur thèse en particulier), selon trois modalités :

- domaine de validité non pris en compte, si l'élève propose son argument ou sa thèse comme une vérité générale, sans modalisation ;

- domaine de validité mentionné si la modalisation est générale (« dans certains cas »...);

- domaine de validité spécifié si la modalisation correspond à une situation précise (« dans ce caslà »...).

Par exemple, pour nous, l'argument 1 proposé ne contient pas de prise en compte du domaine de validité : nous comprenons le «certains environnements " comme « les 
A paraître dans la revue : Recherches en Didactique des Sciences et des Technologies.

environnements où on construit » (par opposition à la mer, par exemple). Cette précision n'est pas réutilisée ensuite. À l'inverse, l'argument 2 contient une mention implicite d'un domaine de validité : la construction d'habitations est parfois la cause de destruction d'environnements, mais pas toujours. Les autres causes ou circonstances n'étant pas spécifiées, nous avons donc codé cette intervention comme mentionnant un domaine de validité.

Notons que, suivant des auteurs comme Zohar et Nemet (2002) ou Sandoval et Millwood (2005), nous avions initialement incorporé à notre grille d'analyse une étude du contenu des arguments, en les évaluant au regard des connaissances stabilisées et reconnues comme non problématiques par l'institution ou des valeurs ou croyances communes. Cette catégorie s'étant révélée inadaptée, nous n'évoquerons pas cette catégorie dans les résultats.

\subsubsection{Indicateurs spécifiques aux Questions Socio-Scientifiques}

Nous présentons maintenant des indicateurs de la qualité argumentative qui sont spécifiques aux QSS.

Nous avons recensé, dans les débats et les tests les différents domaines de la QSS mobilisés, un argument pouvant mettre en interaction plusieurs domaines. Pour cela, nous avons distingué les huit domaines suivants (en nous inspirant de Simonneaux \& Simonneaux, 2011) :

- le domaine scientifique, qui renvoie à des aspects théoriques relevant d'une discipline de recherche, par exemple à des modèles ou à la faisabilité théorique d'une action ;

- le domaine technique, qui renvoie à des aspects pratiques mettant en jeu des techniques ou des technologies, par exemple, qui concerne les moyens mis en œuvre ou la faisabilité matérielle d'une action ;

- le domaine social, renvoyant aux aspects socio-économiques, socio-culturels, et les pratiques sociales ;

- le domaine économique, qui est plus spécifique à l'économie globale d'un pays ou d'une entreprise ;

- le domaine politique, qui correspond aux choix de gouvernance, aux décisions à grande échelle ou aux législations ;

- le domaine axiologique, qui renvoie aux valeurs morales, éthiques et/ou religieuses ;

- le domaine sanitaire, qui renvoie aux considérations sur la santé humaine, individuelle ou publique ;

- le domaine environnemental enfin, qui renvoie aux rapports entre l'être humain et son milieu ainsi qu'aux problématiques de préservation de l'environnement.

Ainsi, les deux arguments présentés dans la figure 5 renvoient aux rapports entre être humain et environnement (" détruire des endroits " pour construire des infrastructures, affectant la biodiversité) mais aussi aux pratiques sociales et socio-économiques (avoir une habitation, raisons autres que la construction d'habitations pour " détruire des endroits "). Ils ont ainsi été codés comme relevant à la fois des domaines environnemental et social. Notons qu'il est possible que certaines interventions ne puissent pas être rattachées à un domaine, notamment dans le cas d'interventions trop vagues ou « vides » (par exemple « ok d'accord »). 
A paraître dans la revue : Recherches en Didactique des Sciences et des Technologies.

Par ailleurs, nous avons renseigné la prise en compte des incertitudes relatives aux connaissances mobilisées, comme celles relatives aux possibilités de développements technologiques ou d'évolutions environnementales ou sociologiques (Morin etal., 2014; Morin, Simonneaux \& Tytler, 2017). Par exemple, aucun des deux arguments présentés dans la figure 5 ne contient de mention d'incertitude. En revanche, l'argument (issu d'un débat sur la voiture électrique) « Les scientifiques travaillent aussi sur des biocarburants pour des moteurs thermiques tout en utilisant une énergie propre. " évoque les possibles développements futurs des sciences et technologies; ainsi, nous considérons qu'il contient une mention des incertitudes relatives à la question.

\subsection{Analyse macro de la structure des débats}

Après avoir effectué une analyse de chaque argument (que nous appelons « analyse micro »), nous avons réutilisé le codage des arguments selon la modalité « Domaines de la QSS » pour mener une analyse au niveau macroscopique de la structure des débats. Au moyen du logiciel XMind, nous avons développé la représentation de la structure des débats générée par la plateforme AREN en annotant chaque intervention selon les domaines de la QSS mobilisés. Nous parlons dans la suite de ces représentations comme des " cartes " des débats. Ces cartes arborescentes sont constituées de différentes " branches », qui représentent des suites d'arguments initiées par le texte et s'enchaînant de façon linéaire. À titre d'exemple, dans la figure 3 ci-dessus et en considérant que l'intervention de Quentin (à gauche) se réfère au texte, on peut compter, de haut en bas, des branches de "longueur » respectives 6, 6, 7, 5 et 5 (la longueur correspondant aux nombres d'interventions successives d'une branche).

Pour la constitution de ces cartes, contrairement à l'analyse micro, nous avons conservé les interventions de type « bavardage » quand elles donnaient lieu à au moins une réponse à visée argumentative (4 interventions concernées), pour pouvoir conserver toutes les interventions argumentatives. Nous n'avons ainsi supprimé les bavardages que dans le cas de branches entièrement non-argumentatives ou dans le cas où ces bavardages se situaient à la fin des branches.

La structure des débats permet de cerner dans quelle mesure les élèves se sont approprié l'outil et le texte. Une fois les cartes constituées, nous avons relevé le nombre de branches de chaque longueur, que nous avons rapporté au nombre de branches total. En outre, nous avons relevé d'où provenaient les différentes branches par rapport au texte support du débat, afin d'évaluer a posteriori le potentiel de chacun des textes.

Une partie de l'analyse a été motivée par le caractère socio-scientifique des débats étudiés. Nous avons ainsi recensé le nombre de domaines présents dans chaque branche et rapporté ce nombre à la longueur de la branche. Cela nous permet d'évaluer dans quelle mesure les élèves articulent plusieurs domaines dans un même échange.

Afin de déterminer la manière dont les élèves articulent les domaines de la QSS au cours du débat, nous avons distingué les dimensions monologale et dialogale : d'une part, nous avons regardé dans chaque intervention individuelle si des domaines étaient articulés, d'autre part, nous 
A paraître dans la revue : Recherches en Didactique des Sciences et des Technologies.

avons regardé au sein de chaque branche du débat, dans laquelle au moins deux élèves sont intervenus, si plusieurs domaines étaient mobilisés et articulés. Au niveau des interventions individuelles, nous avons compté le nombre d'interventions associant plusieurs domaines dans chaque débat. Au niveau des branches du débat, nous avons recensé le nombre de fois où émergeait dans une branche un domaine qui n'y était pas précédemment, c'est-à- dire ni dans l'intervention « racine » ni dans toutes celles qui ont précédé (dans la branche) l'intervention en question. Par exemple, nous pouvons repérer l'émergence d'un domaine dans la branche « Social $\rightarrow$ Social $\rightarrow$ Social/Politique » (où apparaît le domaine politique dans le troisième argument), et deux domaines dans la branche « Scientifique/Technique $\rightarrow$ Social $\rightarrow$ Social/Économique $\rightarrow$ Technique/Économique » (où apparaissent le domaine social au second argument et le domaine économique au troisième).

\section{Résultats}

Comme nous l'avons déjà signalé, notre échantillon est constitué d'une diversité de classes, et ce, non pas dans une perspective comparatiste, mais dans l'objectif d'explorer les potentialités de la plateforme AREN dans un panel assez large de situations. Les différences entre les classes sont liées à de multiples variables : niveau scolaire et âge, milieu socio-culturel, filière, discipline, sujet de débat et texte à débattre. De ce fait, l'échantillon ne permet pas de faire de comparaison entre les classes. Néanmoins, la prise en compte de ces variables (en particulier, de la discipline) peut être pertinente dans certains cas au regard de nos questions de recherche. Dans ce qui suit, les résultats sont donnés sous forme de pourcentages et les nombres entre parenthèses correspondent aux effectifs. Nous présentons tout d'abord les résultats de l'analyse micro (caractère socio-scientifique et qualité de l'argumentation), puis les résultats de l'analyse macro (structure des débats).

\subsection{Analyse micro des débats}

Sur les 10 débats analysés (953 interventions au total), les élèves ont contribué de façon très diverse, avec des débats allant de 20 à 186 interventions (bavardages exclus). Notons que la quasi-totalité des élèves présents ont pris part aux débats, avec un nombre d'interventions très variable (de une à plus de vingt interventions par élève, en l'espace d'environ une heure).

\subsubsection{Indicateurs liés au caractère socio-scientifique des débats}

Le tableau 1 présente le nombre de débats abordant entre 2 et 7 domaines de manière relativement importante (plus de $10 \%$, plus de $5 \%$ ). Comme on peut le voir dans ce tableau, la plupart des dimensions de la QSS sont abordées par les élèves : dans 8 débats, entre 3 et 5 domaines différents sont traités dans au moins $10 \%$ des interventions. Bien qu'ils ne soient pas traités en profondeur, plusieurs domaines supplémentaires sont abordés (tableau 1) de façon plus marginale (entre 5 et $10 \%$ des interventions). Nous n'avons pas pris en compte les domaines apparaissant dans moins de $5 \%$ des interventions, car nous avons considéré qu'ils n'étaient 
A paraître dans la revue : Recherches en Didactique des Sciences et des Technologies.

traités que de façon anecdotique. On observe une grande variabilité selon les débats (tableau 1). En effet, dans certains débats, les élèves ne traitent en profondeur que deux domaines tout en évoquant de façon périphérique plusieurs autres. Dans d'autres, les élèves traitent en profondeur un plus grand nombre de domaines (jusqu'à cinq).

\begin{tabular}{|c|c|c|}
\hline $\begin{array}{c}\text { Nombre de } \\
\text { domaines abordés } \\
\text { dans le débat }\end{array}$ & $\begin{array}{c}\text { Au-dessus } \\
\text { de } 10 \%\end{array}$ & $\begin{array}{c}\text { Au-dessus } \\
\text { de } 5 \%\end{array}$ \\
\hline 2 & 2 débats & Aucun débat \\
\hline 3 & 1 débat & Aucun débat \\
\hline 4 & 6 débats & 3 débats \\
\hline 5 & 1 débat & 4 débats \\
\hline 6 & Aucun débat & 2 débats \\
\hline 7 & Aucun débat & 1 débat \\
\hline
\end{tabular}

Tableau 1 : nombre de domaines abordés dans les débats

Prenons ici l'exemple de deux débats contrastés, le premier en SVT au niveau seconde, le second en EMC au niveau terminale. Comme on peut le remarquer dans le tableau 2, les domaines traités varient considérablement selon le thème de la séquence et le texte débattu. Par exemple dans le débat de SVT portant sur la biodiversité ${ }^{5}$, le domaine scientifique est très présent (33\%) alors qu'il l'est peu dans le débat d'EMC portant sur l'eugénisme (3,4\%). Par ailleurs, les élèves ont très peu mentionné les incertitudes relatives aux savoirs mis en jeu dans le traitement des QSS, avec seulement 3,5 \% (32) des interventions qui en font mention (maximum : $15 \%$ [3], minimum : [0]).

\begin{tabular}{|c|c|}
\hline $\begin{array}{c}\text { Débat SVT 1 } \\
\text { (42 interventions) }\end{array}$ & $\begin{array}{c}\text { Débat EMC 3 } \\
(147 \text { interventions) }\end{array}$ \\
\hline $\mathbf{4 7 , 6 \%}(20)$ environnemental & $59,9 \%(88)$ social \\
\hline $40 \%(17)$ social & $53,7 \%(79)$ axiologique \\
\hline $33 \%(14)$ scientifique & $6,1 \%(9)$ sanitaire \\
\hline $19 \%(8)$ économique & $6,1 \%(9)$ économique \\
\hline $12 \%(5)$ sanitaire & $5,4 \%(8)$ politique \\
\hline $5 \%(2)$ axiologique & $3,4 \%(5)$ scientifique \\
\hline $2,4 \%(1)$ politique & $2,7 \%(4)$ technique \\
\hline
\end{tabular}

Tableau 2 : répartition des arguments selon les dimensions de la question traitée dans deux débats portant sur des QSS différentes

\footnotetext{
5 Nous rappelons qu'un bref descriptif des thèmes et des textes débattus se trouve en annexes 1 et 2.
} 


\subsubsection{Qualité de l'argumentation déployée}

Dans le tableau 3, nous avons regroupé les résultats par discipline pour étudier d'éventuels effets de la discipline, de la QSS traitée ou du texte sur la qualité argumentative. Nous présentons également une moyenne globale des dix débats, ainsi que les taux minimum et maximum pour chaque item.

Ce tableau montre que les résultats suivant les disciplines sont globalement proches pour l'ensemble des items. Cependant, suivant l'item considéré, les débats dans certaines disciplines apparaissent comme étant de meilleure qualité. Par exemple, les débats en classe d'histoiregéographie ressortent comme comprenant davantage de réfutations de la thèse et de la justification, alors que les débats en classe de SPC se distinguent par le pourcentage important d'arguments intégrant un domaine de validité. Nous ne pouvons donc pas considérer que globalement les débats dans une discipline soient de meilleure qualité. À ce stade de la recherche, il ne nous est pas possible de distinguer l'impact de la discipline de celui du texte débattu. Les taux minimum et maximum par item témoignent d'une grande variabilité entre les débats, mais qui ne se traduisent pas par des différences significatives entre disciplines.

\begin{tabular}{|c|c|c|c|c|c|c|c|c|c|c|c|c|c|c|c|}
\hline \multicolumn{2}{|c|}{$\begin{array}{c}\text { Qualité } \\
\text { argumentative }\end{array}$} & \multicolumn{2}{|c|}{$\begin{array}{l}\text { Moyenne } \\
\text { globale } \\
\text { (953 posts) }\end{array}$} & \multicolumn{2}{|c|}{$\begin{array}{c}\text { SVT } \\
\text { (2 débats, } \\
175 \text { posts) }\end{array}$} & \multicolumn{2}{|c|}{$\begin{array}{l}\text { HG } \\
\text { (2 débats, } \\
86 \text { posts) }\end{array}$} & \multicolumn{2}{|c|}{$\begin{array}{c}\text { SPC } \\
\text { (3 débats, } \\
279 \text { posts) }\end{array}$} & \multicolumn{2}{|c|}{$\begin{array}{c}\text { EMC } \\
\text { (3 débats, } \\
413 \text { posts) }\end{array}$} & \multicolumn{2}{|c|}{ Minimum } & \multicolumn{2}{|c|}{ Maximum } \\
\hline \multicolumn{2}{|c|}{$\begin{array}{l}\text { Réfutation de la } \\
\text { thèse }\end{array}$} & \multicolumn{2}{|c|}{$26 \%(248)$} & \multicolumn{2}{|c|}{$23,4 \%(41)$} & \multicolumn{2}{|c|}{$31,4 \%(27)$} & \multicolumn{2}{|c|}{$21,9 \%(61)$} & \multicolumn{2}{|c|}{$28,8 \%(119)$} & \multicolumn{2}{|c|}{$7 \%(6)$} & \multicolumn{2}{|c|}{$42,5 \%(34)$} \\
\hline \multicolumn{2}{|c|}{$\begin{array}{c}\text { Réfutation de la } \\
\text { justification }\end{array}$} & \multicolumn{2}{|c|}{$14,1 \%(134)$} & \multicolumn{2}{|c|}{$10,3 \%(18)$} & \multicolumn{2}{|c|}{$26,7 \%(21)$} & \multicolumn{2}{|c|}{$15,8 \%(44)$} & \multicolumn{2}{|c|}{$12,3 \%(51)$} & \multicolumn{2}{|c|}{$3,5 \%(3)$} & \multicolumn{2}{|c|}{$35 \%(7)$} \\
\hline \multicolumn{2}{|c|}{ Concession } & \multicolumn{2}{|c|}{$9,8 \%(93)$} & \multicolumn{2}{|c|}{$9,7 \%(17)$} & \multicolumn{2}{|c|}{$15,1 \%(13)$} & \multicolumn{2}{|c|}{$12,9 \%(36)$} & \multicolumn{2}{|c|}{$6,5 \%(27)$} & \multicolumn{2}{|c|}{$4,8 \%(7)$} & \multicolumn{2}{|c|}{$20 \%(4)$} \\
\hline \multicolumn{2}{|c|}{ Nuance } & \multicolumn{2}{|c|}{$14,9 \%(142)$} & \multicolumn{2}{|c|}{$20 \%(35)$} & \multicolumn{2}{|c|}{$8,2 \%(7)$} & $22,9 \%$ & $\%(64)$ & $8,7 \%$ & (36) & 6,25 & $\%(5)$ & $25,6^{\circ}$ & $6(22)$ \\
\hline $\begin{array}{l}\text { Que } \\
\text { ner }\end{array}$ & $\begin{array}{l}\text { tion- } \\
\text { nent }\end{array}$ & 9,8 & (93) & $4,6 \%$ & $\%(8)$ & $4,6^{\circ}$ & (4) & $12,2^{\circ}$ & $\%(34)$ & $11,4 \%$ & $6(47)$ & $2,4^{\circ}$ & $\%(1)$ & $16 \%$ & (29) \\
\hline & $\begin{array}{c}1 \\
\text { raison }\end{array}$ & & $\begin{array}{c}57,2 \% \\
(325)\end{array}$ & & $\begin{array}{c}57,5 \% \\
(73) \\
\end{array}$ & & $\begin{array}{c}45,3 \% \\
(21) \\
\end{array}$ & & \begin{tabular}{|c|}
$50,9 \%$ \\
$(84)$ \\
\end{tabular} & & \begin{tabular}{|c|}
$62,6 \%$ \\
$(139)$ \\
\end{tabular} & & \begin{tabular}{|c|}
$77,5 \%$ \\
$(69)$
\end{tabular} & & $\begin{array}{c}23,5 \% \\
(4)\end{array}$ \\
\hline Justifi- & $\begin{array}{c}2 \\
\text { raisons }\end{array}$ & $\begin{array}{c}59,6 \\
\%\end{array}$ & $\begin{array}{c}25,5 \% \\
(145)\end{array}$ & $72,6 \%$ & $\begin{array}{c}26,8 \% \\
(34)\end{array}$ & $74,4 \%$ & $\begin{array}{c}37,5 \% \\
(24)\end{array}$ & $59,1 \%$ & $\begin{array}{c}25,5 \% \\
(42)\end{array}$ & $53,8 \%$ & $\begin{array}{c}20,3 \% \\
(45)\end{array}$ & $48 \%$ & $\begin{array}{c}12,3 \% \\
(11)\end{array}$ & $85 \%$ & $\begin{array}{c}47,1 \% \\
(8)\end{array}$ \\
\hline & $\begin{array}{c}3 \\
\text { raisons } \\
\text { ou plus }\end{array}$ & & $\begin{array}{c}17,3 \% \\
(98)\end{array}$ & & $\begin{array}{c}15,7 \% \\
(20)\end{array}$ & & $\begin{array}{c}17,2 \% \\
(11)\end{array}$ & & $\begin{array}{c}17,6 \% \\
(29)\end{array}$ & & $\begin{array}{c}17,1 \% \\
(38)\end{array}$ & & $\begin{array}{c}11,2 \% \\
(9)\end{array}$ & & $\begin{array}{c}29,4 \% \\
(5)\end{array}$ \\
\hline $\begin{array}{r}\text { Doma } \\
\text { val } \\
\text { (men } \\
\text { spécif }\end{array}$ & $\begin{array}{l}\text { ine de } \\
\text { dité } \\
\text { ion ou } \\
\text { cation) }\end{array}$ & $15,2^{c}$ & $(145)$ & $19,7 \%$ & $\%(52)$ & $13 \%$ & (11) & $36,8 \%$ & b (103) & $21 \%$ & (87) & 10,8 & $\%(7)$ & $44 \%$ & (32) \\
\hline
\end{tabular}

Tableau 3 : caractérisation de la qualité argumentative des débats 
A paraître dans la revue : Recherches en Didactique des Sciences et des Technologies.

4.2. Analyse macro: structure des débats

Nous avons tout d'abord analysé globalement la structure de chacun des 10 débats. Dans chaque débat, de nombreux passages du texte donnent lieu à des échanges argumentés (entre 9 et 24 selon les débats) : en moyenne 16 passages différents du texte sont sélectionnés. Le nombre de passages du texte mis en débat ne semble pas dépendre du texte débattu ou du niveau scolaire. Notons qu'il ne semble pas non plus y avoir de lien entre le nombre de points de départ du texte étudié et la structure générale des débats ou l'exploration des dimensions la QSS par les élèves ou la classe.

Au niveau de l'exploration individuelle de la QSS, $44 \%$ des interventions (420/953) articulent plusieurs domaines. Parmi celles-ci, 18,5\% (78) articulent trois domaines ou plus. Ces interventions à domaines multiples peuvent se retrouver en réaction au texte ou en réponse à un autre élève, plus loin dans une branche. Concernant le traitement collectif de la QSS par les élèves lors du débat, nous avons relevé 211 émergences de domaines dans des branches qui ne les contenaient pas initialement, soit 21 en moyenne par débat.

Dans le tableau 4, nous présentons pour chaque branche de débat (pour l'ensemble des 10 débats, toutes classes et disciplines confondues) le nombre de domaines de la QSS explorés et la longueur des branches. Pour chacune de ces deux variables, nous indiquons également dans le tableau les pourcentages minimums et maximums obtenus pour chaque branche.

\begin{tabular}{|c|c|c|c|c|c|c|c|c|c|}
\hline & $\underset{1}{\text { Longueur }}$ & $\begin{array}{c}\text { Longueur } \\
2\end{array}$ & $\begin{array}{c}\text { Longueur } \\
3\end{array}$ & $\begin{array}{c}\text { Longueur } \\
4-5\end{array}$ & $\begin{array}{c}\text { Longueur } \\
6-7\end{array}$ & Total & $\begin{array}{l}\text { Fréquence } \\
\text { (en \%) }\end{array}$ & $\begin{array}{c}\text { Fréquence } \\
\text { minimale } \\
\text { (en \%) }\end{array}$ & $\begin{array}{l}\text { Fréquence } \\
\text { maximale } \\
(\text { en \%) }\end{array}$ \\
\hline Pas de domaine & 14 & 1 & 1 & 0 & 0 & 16 & 2,61 & 0 & $11,6(5)$ \\
\hline 1 Domaine & 98 & 62 & 11 & 5 & 0 & 176 & 28,71 & $8,33(5)$ & $48(25)$ \\
\hline 2 Domaines & 104 & 112 & 37 & 10 & 2 & 265 & 43,23 & $25,6(11)$ & $49,1(55)$ \\
\hline 3 Domaines & 23 & 51 & 27 & 10 & 0 & 111 & 18,11 & $3,85(2)$ & $35,9(33)$ \\
\hline 4-5 Domaines & 1 & 16 & 12 & 11 & 5 & 45 & 7,34 & $1,8(2)$ & 30 (18) \\
\hline Total & 240 & 242 & 88 & 36 & 7 & 613 & 100 & & \\
\hline Fréquence (en \%) & 39,15 & 39,48 & 14,36 & 5,87 & 1,14 & 100 & & & \\
\hline $\begin{array}{c}\text { Fréquence } \\
\text { minimale (en \%) }\end{array}$ & $1,67(1)$ & $11,76(2)$ & $2,86(1)$ & 0 & 0 & & & & \\
\hline $\begin{array}{c}\text { Fréquence } \\
\text { maximale (en \%) }\end{array}$ & $82,4(14)$ & $62,5(35)$ & $40(16)$ & $28,33(17)$ & $5(3)$ & & & & \\
\hline
\end{tabular}

Tableau 4 : analyse de la structure des débats en termes de domaines et de longueurs des branches 
A paraître dans la revue : Recherches en Didactique des Sciences et des Technologies.

Comme on peut le voir dans le tableau 4, globalement les branches sont relativement courtes. En effet, on compte presque $40 \%$ de branches de longueur 1, c'est-à-dire constituées d'une intervention basée sur le texte sans réaction d'un autre élève. De même, on trouve une proportion équivalente de branches de longueur 2, c'est-à-dire constituées d'une intervention basée sur le texte suivie d'une seule réponse d'un autre élève. Néanmoins, presque $70 \%$ des branches articulent deux domaines (43\%) ou plus (25\%). De plus, comme on peut le voir en figure 6 , les domaines explorés ne sont pas les mêmes dans les différentes branches. Cela montre que, dans la grande majorité des branches et plus globalement sur l'ensemble des débats, les élèves explorent la complexité des QSS. À noter que ces fréquences globales masquent une très grande variabilité selon les débats, comme l'indiquent les fréquences minimales et maximales.

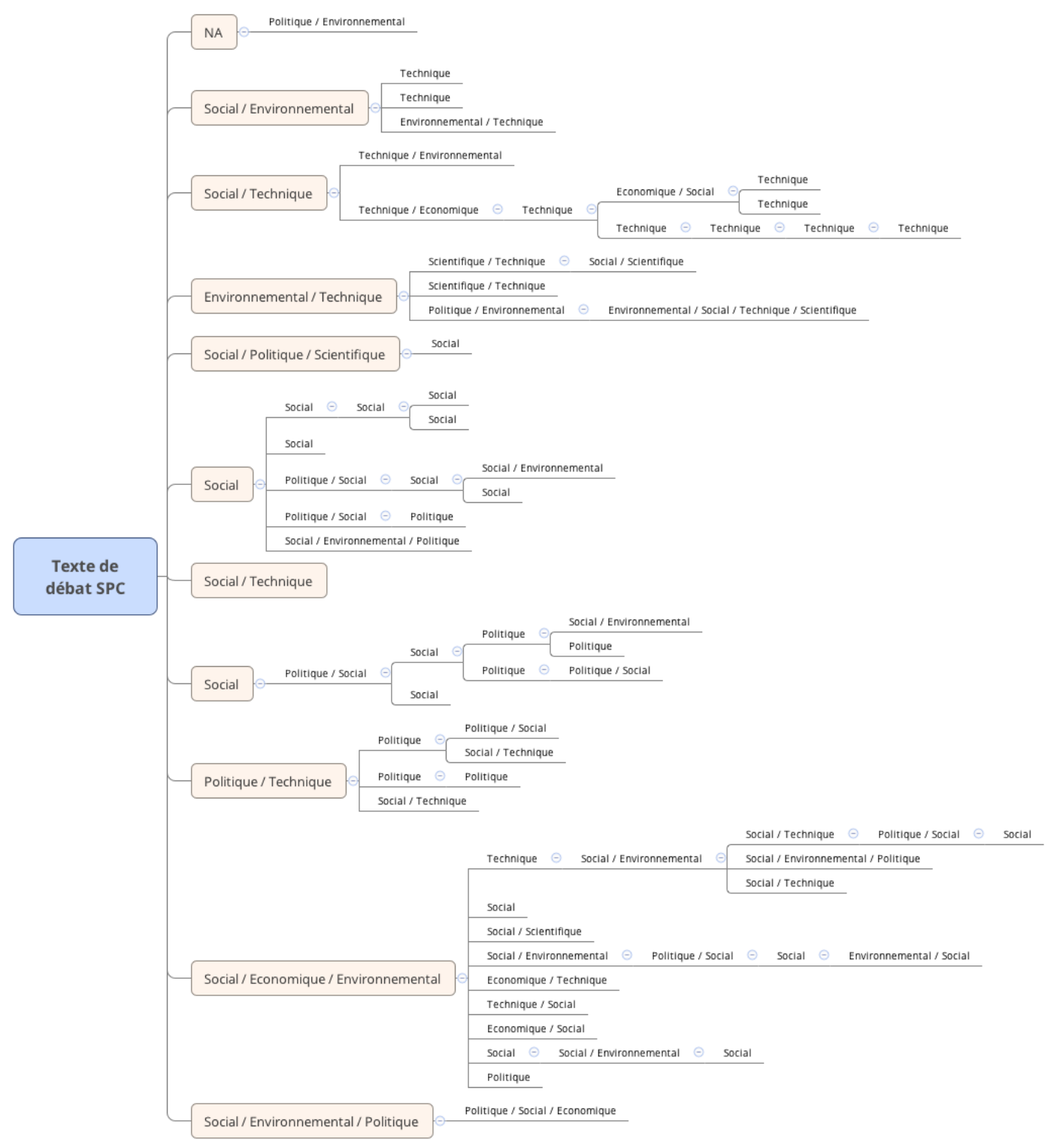

Fig. 6 : carte représentant la structure d'une partie d'un débat 
A paraître dans la revue : Recherches en Didactique des Sciences et des Technologies.

Nous présentons dans le tableau 5 une analyse plus fine de la structure des débats sur deux QSS, l'une traitée en SPC en première $S$ et l'autre en SVT en seconde. On peut y voir des structures de débats très contrastées au niveau de la longueur des branches : les débats en SVT ont été majoritairement composés d'interventions relatives au texte, sans réponse d'un autre élève. II n'y a pas de branche d'une longueur supérieure à 3. À l'inverse, les débats en SPC sont majoritairement constitués de branches de longueur 2, avec presque un quart de branches de longueur 3. En outre, on peut y retrouver des branches de longueur supérieure à 4 en proportion non négligeable, même si la fréquence des branches les plus longues est le plus souvent anecdotique.

En revanche, on peut remarquer que les nombres de domaines mobilisés sont (en proportion ou en pourcentage) assez similaires entre les débats en SVT et en SPC, hormis le taux de branches comprenant 4 ou 5 domaines qui est plus élevé en SPC (ce qui peut s'expliquer par la fréquence plus importante de branches plus longues).

\begin{tabular}{|c|c|c|c|c|c|c|c|}
\hline SPC & $\begin{array}{c}\text { Longueur } \\
1\end{array}$ & $\begin{array}{c}\text { Longueur } \\
2\end{array}$ & $\begin{array}{c}\text { Longueur } \\
3\end{array}$ & $\begin{array}{c}\text { Longueur } \\
4-5\end{array}$ & $\begin{array}{c}\text { Longueur } \\
6-7\end{array}$ & Total & $\begin{array}{c}\text { Fréquence } \\
\text { (en \%) }\end{array}$ \\
\hline Pas de domaine & 0 & 1 & 0 & 0 & 0 & 1 & 0,64 \\
\hline 1 Domaine & 8 & 17 & 6 & 3 & 0 & 34 & 21,66 \\
\hline 2 Domaines & 9 & 31 & 12 & 4 & 0 & 56 & 35,67 \\
\hline 3 Domaines & 0 & 19 & 14 & 6 & 0 & 39 & 24,84 \\
\hline 4-5 Domaines & 0 & 9 & 7 & 8 & 3 & 27 & 17,2 \\
\hline Total & 17 & 77 & 39 & 21 & 3 & 157 & 100 \\
\hline Fréquence (en \%) & 10,83 & 49,04 & 24,84 & 13,38 & 1,91 & 100 & \\
\hline SVT & $\begin{array}{c}\text { Longueur } \\
1\end{array}$ & $\begin{array}{c}\text { Longueur } \\
2\end{array}$ & $\begin{array}{c}\text { Longueur } \\
3\end{array}$ & $\begin{array}{c}\text { Longueur } \\
4-5\end{array}$ & $\begin{array}{c}\text { Longueur } \\
6-7\end{array}$ & Total & $\begin{array}{c}\text { Fréquence } \\
\text { (en \%) }\end{array}$ \\
\hline Pas de domaine & 1 & 0 & 0 & 0 & 0 & 1 & 0,78 \\
\hline 1 Domaine & 20 & 9 & 0 & 0 & 0 & 29 & 22,66 \\
\hline 2 Domaines & 40 & 12 & 5 & 0 & 0 & 57 & 44,53 \\
\hline 3 Domaines & 16 & 14 & 7 & 0 & 0 & 37 & 28,91 \\
\hline 4-5 Domaines & 1 & 1 & 2 & 0 & 0 & 4 & 3,13 \\
\hline Total & 78 & 36 & 14 & 0 & 0 & 128 & 100 \\
\hline Fréquence (en \%) & 60,94 & 28,13 & 10,94 & $\mathbf{0}$ & 0 & 100 & \\
\hline
\end{tabular}

Tableau 5 : nombre de domaines abordés et longueur des branches des débats en SPC et SVT

Les tableaux 4 (fréquences minimum et maximum) et 5 montrent donc une grande variété de structures selon les débats. Cela nous a conduits à proposer une catégorisation de différents " profils de débats ». Cette première catégorisation, loin d'être une typologie exhaustive, sera à 
A paraître dans la revue : Recherches en Didactique des Sciences et des Technologies.

affiner et à mettre à l'épreuve des faits et des données supplémentaires lors des prochaines itérations du dispositif didactique. Ainsi, nous pouvons catégoriser la structure des 10 débats étudiés de la manière suivante :

- les débats " en peigne », très peu interactifs, composés en grande majorité (plus de $75 \%$ ) de branches de longueur 1, comprenant très peu de branches de longueur 3 et aucune branche de longueur supérieure. Parmi les dix débats analysés, deux sont dans cette catégorie ;

- les débats "commentaire de texte collaboratif », avec plus de la moitié de branches de longueur 1 et environ un tiers (plus ou moins $5 \%$ ) de branches de longueur 2. Comme les précédents, les branches de ces débats sont le plus souvent d'une longueur maximale de 3 , même si on peut relever quelques « pics » de longueur 4 à 6 . Quatre débats relèvent de cette catégorie ;

- les débats « buissonnants », avec peu de branches de longueur 1 (de 7 à $16 \%$ ), une majorité de branches de longueur 2 (de 51 à $62 \%$ ) et environ $20 \%$ de branches de longueur 3 . Des branches plus longues sont possibles, bien que rares (au maximum 15\% de branches de longueur supérieure à3). Deux débats relèvent de cette catégorie ;

- les débats "arborescents ", beaucoup plus interactifs. Ici, deux débats relèvent de cette catégorie. Le premier comprend des branches de longueur maximale 4 (10\% des branches) et $17,5 \%$ de branches de longueur 1 , mais avec un très fort taux (40 \%) de branches de longueur 3 et presque un tiers $(32,5 \%)$ de branches de longueur 2 . Le second ne contient lui presque aucune branche de longueur 1 (moins de $2 \%$ ), au profit d'un taux élevé de branches longues ( $25 \%$ de branches de longueurs comprises entre 4 et 7 ). II contient cependant presque la moitié $(46,5 \%)$ de branches de longueur 2 .

\subsection{Analyse des pré/post-tests}

107 élèves ayant suivi l'ensemble du dispositif (phase de préparation, débat et synthèse) ${ }^{6}$ ont passé à la fois le pré-test et le post-test ${ }^{7}$. Nous avons utilisé la même grille d'analyse que pour l'analyse de débat et comparé les taux pour les différents items. Rappelons qu'il s'agissait dans ces tests, pour chaque élève, de se positionner et d'argumenter par écrit sur une affirmation relative à une QSS en lien avec celle débattue. Au niveau de la qualité argumentative, nous n'observons pas de différence significative entre les pré-tests et les post-tests (tableau 6). Nous relevons néanmoins des différences importantes entre la qualité de l'argumentation déployée dans les tests et en débat. Nous considérons que le taux élevé de justifications dans les tests (97\% dans les pré-tests et $93 \%$ dans les post-tests) est probablement induit par la tâche proposée : bien que l'élève argumente face à un interlocuteur virtuel (ce qui rapproche la tâche d'une situation dialogale), elle ou il doit déployer en une fois l'ensemble de son argumentaire. De même, la nature de la tâche peut expliquer le taux très faible de réfutations de la justification $(1,8 \%)$ dans les tests : les assertions par rapport auxquelles les élèves doivent se positionner sont des thèses sans

\footnotetext{
6 Deux classes n'ont pas pu réaliser la phase de synthèse, nous n'analysons donc pas leurs pré et post-tests qui visent à évaluer l'impact du dispositif complet.
}

7 Une classe n'a pas réalisé le post-test. 
A paraître dans la revue : Recherches en Didactique des Sciences et des Technologies.

justification explicite qu'ils pourraient réfuter. La prise en compte par les élèves d'un domaine de validité est bien plus importante dans les pré-tests et les post-tests que lors des débats. En outre, les élèves prennent en compte plus de dimensions de la QSS dans les tests qu'ils ne le faisaient individuellement lors du débat. Le plus souvent, ces dimensions renvoient à des considérations plus " globales » dans les tests que dans les débats (aspects sociaux généraux dans les tests par opposition à l'emploi de valeurs personnelles en débat, par exemple).

\begin{tabular}{|c|c|c|c|c|c|}
\hline & & \multicolumn{2}{|c|}{ Pré-test (107) } & \multicolumn{2}{|c|}{ Post-test (107) } \\
\hline \multicolumn{2}{|c|}{ Réfutation de la thèse } & \multicolumn{2}{|c|}{$39,3 \%(42)$} & \multicolumn{2}{|c|}{$39,3 \%(42)$} \\
\hline \multicolumn{2}{|c|}{ Réfutation de la justification } & \multicolumn{2}{|c|}{$1,8 \%(2)$} & \multicolumn{2}{|c|}{$1,8 \%(2)$} \\
\hline \multicolumn{2}{|c|}{ Concession } & \multicolumn{2}{|c|}{$13,1 \%(14)$} & \multicolumn{2}{|c|}{$9,3 \%(10)$} \\
\hline \multicolumn{2}{|c|}{ Nuance } & \multicolumn{2}{|c|}{$25,2 \%(27)$} & \multicolumn{2}{|c|}{$28 \%(30)$} \\
\hline \multicolumn{2}{|c|}{ Questionnement } & \multicolumn{2}{|c|}{$4,6 \%(5)$} & \multicolumn{2}{|c|}{$1,8 \%(2)$} \\
\hline \multirow{3}{*}{ Justifications } & 1 raison & \multirow{3}{*}{$97,2 \%(104)$} & $22,4 \%(24)$ & \multirow{3}{*}{$93 \%(100)$} & $28 \%(30)$ \\
\hline & 2 raisons & & $37,4 \%(40)$ & & $29,9 \%(32)$ \\
\hline & $\begin{array}{l}3 \text { raisons ou } \\
\text { plus }\end{array}$ & & $37,4 \%(40)$ & & $35,5 \%(38)$ \\
\hline \multicolumn{2}{|c|}{$\begin{array}{l}\text { Domaine de validité (mention ou } \\
\text { spécification) }\end{array}$} & \multicolumn{2}{|c|}{$71 \%(76)$} & \multicolumn{2}{|c|}{$82,2 \%(88)$} \\
\hline \multirow{5}{*}{ Domaines QSS } & Pas de domaine & \multicolumn{2}{|c|}{$0 \%(0)$} & \multicolumn{2}{|c|}{$0,9 \%(1)$} \\
\hline & 1 Domaine & \multicolumn{2}{|c|}{$13,1 \%(14)$} & \multicolumn{2}{|c|}{$26,1 \%(28)$} \\
\hline & 2 Domaines & \multicolumn{2}{|c|}{$56,1 \%(60)$} & \multicolumn{2}{|c|}{$35,5 \%(38)$} \\
\hline & 3 Domaines & \multicolumn{2}{|c|}{$28 \%(30)$} & \multicolumn{2}{|c|}{$28 \%(30)$} \\
\hline & $\begin{array}{l}4 \text { Domaines ou } \\
\text { plus }\end{array}$ & \multicolumn{2}{|c|}{$2,8 \%(3)$} & \multicolumn{2}{|c|}{$9,3 \%(10)$} \\
\hline \multicolumn{2}{|c|}{ Prise en compte de l'incertitude } & \multicolumn{2}{|c|}{$5,6 \%(6)$} & \multicolumn{2}{|c|}{$6,5 \%(7)$} \\
\hline
\end{tabular}

Tableau 6 : résultats des pré-tests et post-tests

\section{Discussion}

Nous discutons à présent les résultats à la lumière de nos deux questions de recherche.

\subsection{Complexité des QSS}

Cette étude, qui s'appuie sur l'analyse de la structure des débats (analyse macro) et des domaines mobilisés dans les arguments (analyse micro), donne à voir les potentialités d'une plateforme numérique pour la mise en œuvre de débats autour de QSS en classe pour développer les compétences argumentatives des élèves sur des QSS et favoriser la prise en compte de leur complexité. La structure arborescente des débats menés sur la plateforme, différente de la linéarité d'un débat oral, permet en effet aux élèves d'explorer simultanément et en parallèle 
A paraître dans la revue : Recherches en Didactique des Sciences et des Technologies.

différentes dimensions d'une QSS et différents aspects de chacune de ces dimensions (par exemple deux interventions relevant du domaine social peuvent se compléter et explorer différentes échelles, retombées possibles, etc.). Les différentes dynamiques des multiples branches du débat témoignent d'un traitement varié de la QSS, dans ses multiples dimensions, donnant ainsi à voir aux élèves sa complexité. Cependant, le mode de débat écrit permis par la plateforme ne garantit pas que les élèves aient lu toutes les interventions : par rapport à un débat oral, où chaque élève entend toutes les interventions, un risque du débat numérique est la potentielle ignorance par certains élèves de pans entiers du débat. Ainsi, il est essentiel d'articuler le débat numérique à une phase de synthèse réflexive permettant aux enseignants de revenir avec les élèves sur la globalité du débat, afin d'en extraire les interventions pertinentes et de revenir sur l'articulation des différentes dimensions de la QSS. Par exemple, la grande majorité des branches mettant en lien deux ou trois domaines, la synthèse permet l'articulation par les élèves de toutes les dimensions de la QSS, toutes traitées lors du débat mais pas nécessairement articulées.

Plus spécifiquement, le fait de partir d'un texte (comme le permet AREN), semble constituer un levier supplémentaire pour favoriser la prise en compte de la complexité des QSS. Tout d'abord, le texte offre des points de départ pour le traitement des multiples dimensions des QSS, comme peuvent le montrer les premières interventions des élèves (figure 6). De plus, dans chaque branche du débat, les élèves ne s'enferment pas nécessairement dans une seule dimension de la QSS : la majorité des branches dans les débats mettent en lien deux ou trois domaines. Chaque branche a ainsi sa dynamique propre d'exploration des différentes dimensions de la QSS et des nouvelles dimensions émergent fréquemment au sein de branches ne les contenant pas a priori. La possibilité d'émergence de domaines au sein de branches du débat et le taux important d'interventions renvoyant à plusieurs domaines semblent donc indiquer que les élèves les articulent lors du traitement du texte, que cela soit collectivement ou individuellement. À titre d'exemple, nous présentons ici un enchaînement de trois interventions trouvant sa source dans le texte de physique (orthographe corrigée). Nous indiquons entre crochets les domaines de la QSS qui sont en jeu ainsi que les critères utilisés pour identifier ces domaines :

Extrait du texte initiateur : "Oui, la voiture de demain sera électrique ».

$F$ : Rien ne permet d'affirmer aujourd'hui que les voitures de demain seront électriques [Technique - emploi d'une technologie spécifique] en raison des nombreux problèmes écologiques que cela engendrerait [Environnemental - conséquences sur l'environnement]. II faudrait régler tous ces problèmes avant de remplacer les moteurs à explosion par des moteurs électriques comme notamment la source d'énergie électrique de demain [Technique - moyens mis en œuvre + Environnemental éventuelle pollution].

Cette intervention met donc en jeu les domaines Environnemental et Technique.

$\mathrm{M}$ : Les voitures de demain se doivent d'être électriques [Politique - décision à grande échelle + Technique - emploi d'une technologie spécifique]. La pollution atmosphérique grandit de jour en jour [Environnemental - pollution] et les voitures, ou du moins les 
A paraître dans la revue : Recherches en Didactique des Sciences et des Technologies.

entreprises qui les construisent (notamment les usines de lithium) ${ }^{8}$, sont la principale source de pollution sur Terre [Environnemental - pollution]. C'est pour cela qu'il faut se diriger vers des énergies plus propres [Politique - décision à grande échelle + Environnemental - pollution] pour remplacer les énergies actuelles trop polluantes.

Cette intervention met donc en jeu les domaines Environnemental et Politique.

K : Oui c'est vrai il faut que la voiture de demain ne soit pas polluante [Technique emploi d'une technologie spécifique (indéterminée) + Environnemental - pollution] mais a l'heure actuelle la voiture électrique est loin de pouvoir assurer les besoins de toute la population [Social - besoin de la population]. Je pense que les chercheurs [Scientifique - recherche] devraient se tourner vers des alternatives écologiques différentes de l'électricité [Technique - faisabilité matérielle + Environnemental]. Car oui la voiture [électrique] ${ }^{9}$ ne pollue pas ou du moins pas énormément [Environnemental pollution] mais le nucléaire qui sert à la fabrication de cette électricité n'est pas sain [Technique - spécificité d'une technique + Environnemental - pollution].

Cette intervention met donc en jeu les domaines Technique, Environnemental, Scientifique et Social.

Comme on peut le voir dans cet exemple, les domaines peuvent être articulés dans des phrases différentes (intervention de $M$ ) ou au sein d'une même phrase (interventions de $F$ et $M$ ). En outre, les élèves peuvent rebondir sur les arguments des autres en faisant émerger de nouveaux domaines au cours de l'échange (interventions de $\mathrm{M}$ et $\mathrm{K}$ ).

Cette dynamique propre aux débats numériques étudiés vient contraster avec d'autres modes de débat, comme le jeu de rôle (Simonneaux, 2003), où les élèves, contraints par le rôle à jouer, ont du mal à explorer individuellement la QSS débattue.

Notons qu'un débat numérique partant d'un texte semble permettre aux élèves de saisir ou faire émerger des questions qui font sens pour eux et dont ils choisissent d'explorer les tenants et les aboutissants, favorisant ainsi la problématisation du débat (au sens de Schwarz \& Baker, 2017, évoqué plus haut), comme le montrent nos résultats concernant la structure des différents débats (notamment le nombre de points de départ du texte, le nombre d'interventions et le caractère plus ou moins arborescent des débats). Partir d'un texte constitue donc une alternative au traitement d'une QSS locale, approche souvent employée pour favoriser la problématisation du débat. Donner aux élèves la possibilité de faire émerger d'un texte les questions qui les interpellent permet ainsi d'être proche de leurs préoccupations, tout en évitant les écueils liés au traitement de questions trop sensibles localement (Simonneaux \& Simonneaux, 2011). La problématisation du débat est, d'après la littérature, une condition nécessaire pour le développement des compétences argumentatives et l'appréhension de la complexité des QSS. II convient donc d'étudier plus avant

\footnotetext{
8 On peut penser que l'élève fait ici référence aux voitures à moteur thermique. La référence aux batteries au lithium, présente dans le texte et visant spécifiquement les voitures électriques, est possiblement une confusion.

9 Pour faciliter la compréhension du lecteur, nous avons ici ajouté le terme « électrique », qui ne figure pas dans l'intervention de l'élève, dans la mesure où ce dernier fait référence sans ambiguïté à la voiture électrique.
} 
A paraître dans la revue : Recherches en Didactique des Sciences et des Technologies.

ce levier, en particulier en tentant d'isoler des caractéristiques des textes qui permettraient à la fois une problématisation pertinente et une argumentation de qualité.

Par ailleurs, la différence de traitement des QSS entre les tests et les débats vient rappeler l'importance de la prise en compte du but et du contexte de l'argumentation (Walton, 1998 ; Garcia-Mila et al., 2013) : lors du débat, même si la consigne était tournée vers l'exploration du texte et de la QSS et la co-construction de connaissances (dans un but de délibération), on peut penser que les élèves ont aussi cherché à se convaincre mutuellement (dans un but de persuasion). Cela a pu avoir un impact sur le choix des arguments avancés par les élèves, et donc possiblement sur les dimensions de la question discutée. En effet, pour convaincre, les élèves ne mobilisent peut-être pas les mêmes arguments que pour explorer une question ou pour défendre leur point de vue. Si l'activité perçue est différente (notamment le fait de s'adresser à un autre élève en débat ou à l'enseignant dans les tests), la production argumentative peut être différente.

Les résultats du post-test montrent que tous les élèves ne remobilisent pas des arguments relevant des multiples domaines mis en jeu lors du débat, ce qui semble indiquer que la synthèse n'a peut-être pas suffisamment permis aux élèves de prendre en compte et d'avoir du recul sur les différentes dimensions de la question. Cela nous amène à envisager des affinements de la phase de synthèse pour les prochaines itérations du dispositif, de manière à favoriser la prise de conscience par les élèves de la complexité des QSS débattues.

\subsection{Qualité de l'argumentation}

Le dispositif a permis une pratique active du débat pour la quasi-totalité des élèves, avec pour certains de très nombreuses interventions. Le débat numérique permet que la participation très active de certains élèves ne se fasse pas au détriment des autres (Schwarz \& Baker, 2017). De ce point de vue, les débats numériques contrastent avec les débats oraux, dans lesquels les enseignants sont amenés à réguler la parole, et où un nombre limité d'élèves ont l'occasion d'intervenir (Simonneaux \& Simonneaux, 2005).

En ce qui concerne la qualité de l'argumentation déployée par les élèves, les tests effectués avant et après la séquence n'ont pas montré d'évolution significative. Il convient toutefois d'être prudents sur l'interprétation de ce résultat. En effet, comme nous l'avons indiqué dans la partie "Résultats ", le contexte des tests (situation monologale) diffère du contexte des débats (situation dialogale), même si nous avons essayé de minimiser cet écart. II se peut que les élèves aient développé des compétences à argumenter en situation de débat sans que cela ne se traduise par une amélioration de leur qualité argumentative en situation monologale. Cette absence d'évolution significative dans les tests peut aussi être liée à un effet de lassitude des élèves. En effet, les enseignants ont rapporté que ces derniers étaient réticents à reproduire le test écrit (pré et post-tests identiques), de surcroît à l'issue d'un dispositif qui a parfois pu s'étendre sur un temps plus long qu'une séquence classique. De plus, les conditions de passation des post-tests n'ont pas, dans la plupart des cas, été optimales : en fin de séance et sur un temps parfois trop court. En outre, plusieurs auteurs ont rapporté que les effets de dispositifs visant à améliorer la qualité argumentative étaient surtout manifestes sur un temps plus long, les effets de court terme 
A paraître dans la revue : Recherches en Didactique des Sciences et des Technologies.

étant ténus (Kuhn, Hemberger \& Khait, 2016). Nous ne pouvions donc nous attendre à des effets très marqués sur une seule séquence : il semble que pour développer les compétences argumentatives, complexes, il faille un temps plus long permettant d'exposer les élèves à de multiples activités argumentatives. II est néanmoins possible que des phénomènes se soient joués à une plus petite échelle et qu'un autre grain d'analyse que celui que nous avons employé soit requis pour mieux comprendre les effets du dispositif didactique mis en place.

Nous nous appuyons sur ces différents éléments pour poursuivre nos recherches. Rappelons que notre projet de recherche est itératif, les résultats présentés ici étant ceux de la première année. II s'agit à la fois d'affiner le dispositif didactique présenté, de développer la modélisation de l'argumentation et la méthodologie d'analyse des débats et des tests. Pour le dispositif didactique, nous allons ainsi mettre en place deux séquences incluant des débats numériques au cours de l'année dans chaque classe afin de nous permettre d'observer des évolutions sur un temps plus long. Cela nous permettra notamment de mesurer les évolutions entre les deux débats, dans un même contexte dialogal. Nous allons également développer la synthèse réflexive avec un travail plus poussé sur les incertitudes et les valeurs mises en jeu dans le traitement des différentes QSS. En effet, les élèves ont très peu soulevé les incertitudes relatives aux différents savoirs mis en jeu lors du traitement des différentes QSS, que cela soit dans les débats ou dans les tests. Cela laisse supposer que sans un travail explicite spécifique sur les incertitudes, les élèves ne les prennent pas en compte spontanément. Par ailleurs, la plateforme numérique AREN va elle aussi évoluer, sur un plus long terme, ce qui aura également un impact sur le dispositif : des modifications au niveau de l'interface devraient permettre une plus grande lisibilité du débat, facilitant alors la synthèse en aidant les élèves à mieux prendre connaissance de tous les arguments mobilisés lors du débat.

Nous allons en outre remplacer la structure pré-test/post-test par une structure de " prétexte/post-texte » (Baker, 2009a), asymétrique, qui permettra peut-être de réduire la lassitude des élèves, en particulier après deux séquences. Nous allons également contrôler plus spécifiquement les conditions de passation des tests: outre les effets de lassitude et les différentes améliorations requises par notre dispositif, il est possible que certains effets aient été masqués par le temps de passation des tests. En effet, certains élèves ont produit des textes plus courts dans le post-test que dans le pré-test, et ne pouvaient donc de fait pas mobiliser dans leur argumentaire autant de dimensions de la QSS traitée.

Enfin, nous poursuivons notre travail de modélisation de l'argumentation, en appui sur la littérature et sur cette première expérimentation, ce qui va nous amener à faire évoluer la grille d'analyse. Pour mieux comprendre les processus complexes de développement des compétences argumentatives des élèves, nous allons de plus compléter cette analyse quantitative avec une analyse qualitative plus fine s'appuyant sur des entretiens avec quelques élèves. 
A paraître dans la revue : Recherches en Didactique des Sciences et des Technologies.

\section{Bibliographie}

ALBE V. (2008). When scientific knowledge, daily life experience, epistemological and social considerations intervene: students' argumentation in group discussions on a socio-scientific issue. Research in Science Education, n³8, p. 67-90.

AUFSCHNAITER C. von, ERDURAN S., OSBORNE J., \& SIMON S. (2008). Arguing to Learn and Learning to Argue: Case Studies of How Students' Argumentation Relates to Their Scientific Knowledge. Journal of Research in Science Teaching, vol. 45, n¹, p. 101-131.

BAKER M.J. (2009a). Intersubjective and intrasubjective rationalities in pedagogical debates: Realizing what one thinks. In B. Schwarz, T. Dreyfus \& R. Hershkowitz (éd.), Transformation of Knowledge Through Classroom Interaction, p. 145-158. Londres : Routledge.

BAKER M.J (2009b) Argumentative Interactions and the Social Contruction of Knowledge. In N.

Muller-Mirza \& A.-N. Perret-Clermont (éd.), Argumentation and Education: Theoretical Foundaions and Practices, Berlin : Springer, p. 127-144.

BEAUFORT S., CAUSSIDIER C., HAGĖGE H., HAUSBERGER B., HAUSBERGER T., MOLINATTIG. \& ROBERTJ.-P. (2015). Organiser un débat en classe sur une question scientifique socialement vive : pourquoi et comment ? Bulletin de l'APBG, p. 85-104.

BRETON P. (2000). Citoyenneté et enseignement de l'argumentation. In Actes des Premières Rencontres inter-IUFM.

BROSSAIS E., PANISSAL N. \& GARCIA-DEBANC C. (2013) Analyses plurielles d'un débat entre élèves. Émergence d'une méthode d'analyse thématico-argumentative. Cahiers de recherche sociologique, $n^{\circ} 54$, p. 113-140.

CE (Commission européenne) (2012). Responsible Research and Innovation: Europe's ability to respond to societal challenges. European Union.

CLARK D. B., STEGMANN K., WEINBERGER A., MENEKSE M. \& ERKENS G. (2007). Technology-Enhanced Learning Environments to Support Students' Argumentation. In S. Erduran \& M. Jiménez-Aleixandre (éd.), Argumentation in Science Education, Berlin : Springer Science/Business Media B.V, p. 217-243.

CLARK D. B. \& SAMPSON V. (2008). Assessing Dialogic Argumentation in Online Environments to Relate Structure, Grounds, and Conceptual Quality. Journal of Research in Science Teaching, vol. 45, $n^{\circ} 3$, p. 293-321.

COBB P., CONFREY J., LEHRER, R. \& SCHAUBLE L. (2003). Design Experiments in Educational Research. Educational Researcher, vol. 32, n`1, p. 9-13.

DRIVER R., NEWTON P. \& OSBORNE J. (2000). Establishing the Norms of Scientific Argumentation in Classrooms. Science Éducation, vol. 84, n³, p. 287-312.

ERDURAN S. (2007). Methodological Foundations in the Study of Argumentation in Science Classrooms. In S. Erduran \& M. Jiménez-Aleixandre (éd.), Argumentation in Science Education, Berlin : Springer Science/Business Media B.V, p. 47-69.

ERDURAN S. (2018). Toulmin's argument pattern as a " horizon of possibilities » in the study of argumentation in science education. Cultural Studies of Science Education, vol. 13, n4, p. 1091-1099. 
A paraître dans la revue : Recherches en Didactique des Sciences et des Technologies.

ERDURAN S., SIMON S. \& OSBORNE J. (2004). TAPping into Argumentation: Developments into the Application of Toulmin's Argument Pattern for Studying Science Discourse. Science Éducation,vol. 88, $n^{\circ}$ 6, p. 915-933.

ERDURAN S. \& JIMENÉZ-ALEIXANDRE M. (2008). Foreword, In S. Erduran \& M. JiménezAleixandre (éd.), Argumentation in Science Education, Berlin : Springer Science/Business Media B.V, p. ix-xv.

ERDURAN S., OZDEM Y. \& PARK J.-Y. (2015). Research trends on argumentation in science education: a journal content analysis from 1998-2004. International Journal of STEM Education, $\mathrm{n}^{\circ} 2$, article 5 .

FELTON M. (2004). The development of discourse strategies in adolescent argumentation. Cognitive Development, vol. 19, n³, p. 5-52.

GAUSSEL M. (2016). Développer l'esprit critique par l'argumentation : de l'élève au citoyen. Dossiers de veille de l'IFÉ, $n^{\circ} 108$, Lyon : ENS de Lyon. En ligne : <http://veille-etanalyses.ens- Iyon.fr/DA/detailsDossier.php?parent=accueil\&dossier=108\&lang=fr $>$.

GARCIA-MILA M. \& ANDERSEN C. (2007). Cognitive Foundations of Learning Argumentation. In S.Erduran \&M.Jiménez-Aleixandre (éd.), Argumentation in Science Education, Berlin : Springer Science + Business Media, p. 29-45.

GARCIA-MILA M., GILABERT S., ERDURAN S. \& FELTON M. (2013). The Effect of Argumentative

Task Goal on the Quality of Argumentative Discourse. Science Éducation, vol. 88, $\mathrm{n}^{\circ} 6, \mathrm{p}$. 915-933.

GOLDER C. \& FAVART M. (2003). Argumenter c'est difficile... Oui, mais pourquoi ? Approche psycholinguistique de la production argumentative en situation écrite. Ela - Études de linguistique appliquée, $n^{\circ} 130$, p. 187-209.

GRECO-MORASSO S., MISEREZ-CAPEROS C. \& PERRET-CLERMONT A.-N. (2015). L'argumentation à visée cognitive chez les enfants. Une étude exploratoire sur les dynamiques argumentatives et psychosociales. In N. M. Mirza \& C. Buty (éd.), L'argumentation dans les contextes de l'éducation, Berne : Peter Lang, p. 39-82.

HABERMAS J. (2001) Vérité et justification. Paris : Gallimard.

HENSERSON J.B., McNEILL K.L., GONZÁLEZ-HOWARD M., CLOSE K. \& EVANS M. (2018). Key Challenges and Future Directions for Educational Research on Scientific Argumentation. Journal of Research in Science Teaching, vol. 55, n¹, p. 5-18.

JIMĖNEZ-ALEIXANDRE M. P. \& ERDURAN S. (2007). Argumentation in Science Education: An Overview. In S. Erduran \& M. Jiménez-Aleixandre (éd.), Argumentation in Science Education Springer, Berlin : Science + Business Media, p. 3-27.

JIMĖNEZ-ALEIXANDRE M., EIXERAS F. \& AGRASO M. F. (2006). Use of evidence in arguments about a socio-scientific issue by $12^{\text {th }}$ grade students: choices about heating system and energy sources. Actes de la réunion de la National Association for Research in Science Teaching.

KELLYG. J. \& TAKAO A. (2002). Epistemic Levels in Argument: An Analysis of University Oceanography Students' Use of Evidence in Writing. Science Éducation, vol. 86, n³, p. 314-342. 
A paraître dans la revue : Recherches en Didactique des Sciences et des Technologies.

KNIPPELS M.-C., VAN DAM F. \& VANHARSKAMP M. (éd.) (2017). Science and society in education: socio-scientific inquiry-based learning, connecting formal and informal science education with society, PARRISE.

KUHN D., SHAW V. \& FELTON M. (1997). Effects of Dyadic Interaction on Argumentative Reasoning. Cognition and Instruction, vol. 15, $n^{\circ} 3$, p. 287-315.

KUHN D. \& UDELL W. (2003). The development of argument skills. Child Development, vol.74, $\mathrm{n}^{\circ} 5$, p. $1245-1260$.

KUHN D., HEMBERGER L. \& KHAIT V. (2016). Argue with me: developing thinking and writing through dialog. Bronxville, NY : Wessex Press.

LANGE J.-M. \& VICTOR P. (2006). Didactique curriculaire et « éducation à... la santé, l'environnement et au développement durable »: quelles questions, quels repères ? Didaskalia, $\mathrm{n}^{\circ} 28$, p. 85-100.

LHOSTE Y. (2006). La construction du concept de circulation sanguine en $3^{\mathrm{e}}$ Problématisation, argumentation et conceptualisation dans un débat scientifique. Aster, n42, p. 79-108.

MACAGNO F. (2015). Argument relevance and structure. Assessing and developing student's uses of evidence. International Journal of Educational Research, vol. 19, n¹, p. 180-194.

MEN (ministère de l'Éducation nationale) (2015). Socle commun de connaissances, de compétences et de culture. Bulletin officiel de l'Éducation nationale, n¹7 du 23 avril 2015.

MORIN O., SIMONNEAUX L., SIMONNEAUX J., TYTLER R. \& BARRAZA L. (2014). Developing and Using and $S^{3} R$ Model to Analyze Reasoning in Web-Based Cross- National Exchanges on Sustainability. Science Éducation, vol. 98, n³, p. 517-542.

MORIN O., SIMONNEAUX, L. \& TYTLER R. (2017). Engaging With Socially Acute Questions: Development and Validation or and Interactional Reasoning Framework. Journal of Research in Science Teaching, vol. 54, $\mathrm{n}^{\circ}$ 7, p. 825-851.

ORANGE C., LHOSTE Y. \& ORANGE-RAVACHOL D. (2008). Argumentation, problématisation et construction de concepts en classe de sciences. In C.Buty \& C. Plantin (éd.). Argumenter en classe de sciences. Du débat à l'apprentissage, Lyon : INRP.

OSBORNE, J., ERDURAN S. \& SIMON S. \& MONK M. (2001). Enhancing the quality of argument in school science. School Science Review, vol. 82, n³01, p. 63-70.

OSBORNE, J., ERDURAN S. \& SIMON S. (2004). Enhancing the Quality of Argument in School Science. Journal of Research in Science Teaching, vol. 41, n¹0, p. 994-1020.

PLANTIN C. (2016). Dictionnaire de l'argumentation. Une introduction aux études d'argumentation, Lyon : ENS Éditions.

POLO C. (2014). L'eau à la bouche : ressources et travail argumentatifs des élèves lors de débats socio-scientifiques sur l'eau potable. Lyon : université Lumière Lyon 2.

REVERDY C. (2016). La coopération entre élèves : des recherches aux pratiques. Dossier de veille de I'IFÉ $n^{\circ} 114$, Lyon : ENS de Lyon. En ligne : <http://veille-et-analyses.ens- Iyon.fr/ DA/detailsDossier.php?parent=accueil\&dossier=114\&lang=fr $>$.

SADLER T. D. \& ZEIDLER D. L. (2005). Patterns of Informal Reasoning in the Context of Socioscientific Decision Making. Journal of Research in Science Teaching, vol. 42, $\mathrm{n}^{\circ} 1, \mathrm{p}$. $112-138$. 
A paraître dans la revue : Recherches en Didactique des Sciences et des Technologies.

SADLER T. D. \& FOWLER S. R. (2006). A Threshold Model of Content Knowledge Transfer for Socioscientific Argumentation. Science Éducation, vol. 90, n6, p. 986- 1004.

SANDOVAL W. (2013). Educational design research in the $21^{\text {st }}$ century. In R. Luckin, J. Underwood, N. Winters, P. Goodyear, B. Grabowski \& S. Puntambekar (éd.), Handbook of design in educational technology, Londres : Taylor \& Francis, p. 388-396.

SANDOVAL W. A. \& MILLWOOD K. A. (2005). The Quality of Students' Use of Evidence in Written Scientific Explanations. Cognition and Instruction, vol. 23, n¹, p. 23-55.

SCHWARZ B. \& BAKER M. (2015). Sur l'adéquation des théories de l'argumentation aux sciences de l'apprentissage et les fondements d'une théorie de "l'argumentissage ». In N. Muller Mirza, C. Buty, (éd.), L'argumentation dans les contextes de l'éducation, Berne : Peter Lang.

SCHWARZ B. \& BAKER M. (2017). Argumentation, Dialogue and Éducation: History, Theory and Practice. Cambrige : Cambridge University Press.

SIMONNEAUX L. (2003). L'argumentation dans les débats en classe sur une technoscience controversée. Aster, n³7, p. 189-214.

SIMONNEAUX L. (2007). Argumentation in Socio-Scientific Contexts. In S. Erduran \& M. JiménezAleixandre (éd.), Argumentation in Science Education. Berlin : Springer Science + Business Media, p. 179-199.

SIMONNEAUX L. \& SIMONNEAUX J. (2005). Argumentation sur des questions socioscientifiques. Didaskalia, n²7, p. 79-108.

SIMONNEAUX L. \& SIMONNEAUX J. (2011). Argumentations d'étudiants sur des Questions socialement vives environnementales. Formation et pratiques d'enseignants en question, $n^{\circ} 13$, p. 157-178.

TOULMIN S. (1958). The Uses of Argument. Cambridge University Press.

WALTON D. (1998). The new dialectic: Conversational contexts of argument. Toronto : University of Toronto Press.

WALTON D. (2010). Why Fallacies Appear to be Stronger Arguments than They Are. Informal Logic, vol. $30, n^{\circ} 2$, p. 159-184. 30

WEISSER M., MASCLET E. \& RÉMIGNY M. (2003). Construction de la compréhension par l'argumentation orale en sciences. Expérience menée au cycle 3. Aster, n³7, p. 17-52.

ZEIDLER D.L., LEDERMANN. G. \& TAYLORS. C. (1992). Fallacies and Student Discourse: Conceptualizing the Role of Critical Thinking in Science Education. Science Education, vol. $76, n^{\circ} 4$, p. $437-450$.

ZEIDLER D.L. (1997) The Central Role of Fallacious Thinking in Science Education. Science Education, vol. 81, n4, p. 483-496.

ZOHAR A. \& NEMET F. (2002). Fostering Student's Knowledge and Argumentation Skills Through Dilemmas in Human Genetics. Journal of Research in Science Teaching, vol. 39, n¹, p. 35-62. 


\section{Annexes}

Annexe 1 - Rapide descriptif des thèmes traités pendant les séquences et analyse a priori des domaines en jeu dans les différentes QSS

\begin{tabular}{|c|c|c|c|c|}
\hline Thèmes & $\begin{array}{l}\text { Classe } \\
\text { et disci- } \\
\text { pline }\end{array}$ & Domaines principaux & $\begin{array}{l}\text { Domaines } \\
\text { secondaires }\end{array}$ & $\begin{array}{l}\text { Domaines plus } \\
\text { périphériques }\end{array}$ \\
\hline $\begin{array}{l}\text { Lien entre } \\
\text { disparition de } \\
\text { la biodiversité } \\
\text { et activité } \\
\text { humaine } \\
\text { (agriculture, } \\
\text { développe- } \\
\text { ment) }\end{array}$ & $\begin{array}{l}2^{\text {nde }} \\
\text { SVT }\end{array}$ & $\begin{array}{l}\text { Environnemental } \\
\text { Scientifique (Notion } \\
\text { de biodiversité) } \\
\text { Social (Organisation } \\
\text { de société) }\end{array}$ & $\begin{array}{l}\text { Politique } \\
\text { Économique } \\
\text { (Régulation ou non } \\
\text { des activités) } \\
\text { Sanitaire (impact de la } \\
\text { pollution sur la santé } \\
\text { humaine) }\end{array}$ & $\begin{array}{l}\text { Technique (Actions de } \\
\text { l'humain) } \\
\text { Axiologique (Liens } \\
\text { avec l'éthique animale) }\end{array}$ \\
\hline $\begin{array}{l}\text { Exploitation } \\
\text { des « gaz de } \\
\text { schiste" }\end{array}$ & $\begin{array}{l}2^{\text {nde }} \\
\text { Géogra } \\
\text { phie }\end{array}$ & $\begin{array}{l}\text { Economique (création } \\
\text { d'emploi et de valeur) } \\
\text { Social (retombées } \\
\text { économiques) } \\
\text { Environnemental } \\
\text { (possibles pollutions) }\end{array}$ & $\begin{array}{l}\text { Politique } \\
\text { (réglementations, } \\
\text { demande de permis ou } \\
\text { d'arrêtés) } \\
\text { Technique (extraction } \\
\text { et combustions moins } \\
\text { polluantes) }\end{array}$ & $\begin{array}{l}\text { Scientifique } \\
\text { Sanitaire (Pollution et } \\
\text { santé humaine) } \\
\text { Axiologique (Liens } \\
\text { avec l'éthique animale) }\end{array}$ \\
\hline $\begin{array}{l}\text { Développe- } \\
\text { ment de } \\
\text { l'usage de } \\
\text { voitures } \\
\text { électriques }\end{array}$ & $\begin{array}{l}1 \mathrm{eS} \\
\mathrm{SPC}\end{array}$ & $\begin{array}{l}\text { Environnemental } \\
\text { (voitures sans } \\
\text { émissions polluantes) } \\
\text { Social (rôle de la } \\
\text { voiture dans la société) } \\
\text { Technique } \\
\text { (composition de la } \\
\text { batterie, mode de } \\
\text { production d'électricité) }\end{array}$ & $\begin{array}{l}\text { Economique (marché } \\
\text { de l'automobile) } \\
\text { Politique (incitations } \\
\text { par des primes ou } \\
\text { majorations) } \\
\text { Sanitaire (impact de la } \\
\text { pollution sur la santé } \\
\text { humaine) }\end{array}$ & $\begin{array}{l}\text { Scientifique (modes } \\
\text { théoriques de } \\
\text { production d'électricité) } \\
\text { Axiologique }\end{array}$ \\
\hline $\begin{array}{l}\text { Sélection des } \\
\text { embryons : } \\
\text { intérêts et } \\
\text { dérives }\end{array}$ & $\begin{array}{l}\text { T STMG } \\
\text { et } \\
\text { STI2D } \\
\text { EMC }\end{array}$ & $\begin{array}{l}\text { Axiologique } \\
\text { (eugénisme, } \\
\text { avortement...) } \\
\text { Social (impact d'une } \\
\text { sélection des } \\
\text { embryons sur la } \\
\text { société) } \\
\text { Sanitaire (dépistage } \\
\text { ou élimination de } \\
\text { maladies génétiques) }\end{array}$ & $\begin{array}{l}\text { Politique } \\
\text { (réglementations) } \\
\text { Technique (différentes } \\
\text { techniques, différentes } \\
\text { possibilités et coûts) }\end{array}$ & $\begin{array}{l}\text { Scientifique (risques } \\
\text { théoriques) } \\
\text { Economique } \\
\text { (retombées } \\
\text { économiques, potentiel } \\
\text { marché) } \\
\text { Environnemental } \\
\text { (sélection animale et } \\
\text { dispersion dans } \\
\text { l'environnement) }\end{array}$ \\
\hline
\end{tabular}


Annexe 2 - Descriptif des textes de débat

SVT - La biodiversité qui dérange. Extrait du livre La biodiversité au quotidien : Le développement durable à l'épreuve des faits, Christian Lévêque.

Ce texte nuancé vise à questionner une vision de la biodiversité comme "victime innocente des méfaits de l'espèce humaine ", en rappelant les avantages que les humains ont tiré d'une limitation volontaire de la biodiversité : par exemple, les pesticides ont été élaborés pour protéger les cultures humaines des agressions extérieures. Le texte aborde principalement les dimensions environnementale, sociale, sanitaire et technique. La dimension politique est également évoquée.

Géographie - Oui, les gaz de schistes peuvent contribuer au redressement productif ! Communiqué de l'Amicale des foreurs et des métiers du pétrole, édité le 14/09/2012.

https://www.euro-petrole.com/oui-les-gaz-de-schiste-peuvent-contribuer-au-redressementproductif-!-n-f-6583, consulté le 17 juillet 2018

Ce texte a pour objectif de présenter aux élèves une vision très tranchée mais bien argumentée en faveur des gaz de schiste. Les dimensions économique et sociale sont fortement mises en avant, ainsi que la dimension environnementale. Les aspects politiques et techniques sont également évoqués.

SPC - La voiture électrique, ou l'arbre qui cache la forêt..., Pierre Japhet, Greenwatchers, octobre 2010.

Le texte vise à questionner la voiture électrique dans son rôle de " remplaçante » de la voiture thermique. Le point de vue développé dans le texte, nuancé, est celui d'une limitation importante de l'usage de la voiture, électrique ou non. Sont principalement évoquées les dimensions environnementale, sociale, technique, économique et politique. Les aspects sanitaires sont absents du texte.

EMC - Les dangers du laisser-faire. Amy Otchet, le Courrier de l'UNESCO, Septembre 1999. p 27-28

Le texte vise à souligner les dérives possibles d'un " eugénisme du laisser-faire ", qui pourrait s'insinuer dans les sociétés mettant en place une sélection des embryons sans la réglementer. Le texte, nuancé, présente les points de vue argumentés de différents acteurs (généticiens, médecins, philosophes, juristes). Sont principalement abordées les dimensions sociale, axiologique, sanitaire, politique et économique.

Annexe 3 - Exemple d'un texte à débattre. Débats « Liens entre disparition de la biodiversité et activité humaine ». Extrait du livre La biodiversité au quotidien: Le développement durable à l'épreuve des faits, Christian Lévêque

La biodiversité renvoie à l'environnement de l'homme, au cadre de vie dans lequel il agit, ou à celui dans lequel il cherche à se détendre. Les ONG et beaucoup de scientifiques dénoncent à juste titre la disparition des espèces et des écosystèmes et réclament des mesures de protection. Ou, pour le moins, un meilleur équilibre entre l'utilisation des ressources vivantes et leur préservation à long terme. En caricaturant, le discours sur la conservation tend cependant à globaliser la biodiversité qui devient la victime innocente des méfaits de l'espèce humaine. Si la biodiversité disparaît c'est à cause des hommes. On parle d'habitats dégradés et d'espèces disparues ou en danger d'extinction. On demande toujours plus d'aires protégées. En bref, le discours souvent culpabilisateur est centré sur la préservation et la conservation des espèces et des écosystèmes.

[...]

Des tigres aux loups, des crocodiles aux piranhas, des hippopotames aux sangliers, de nombreux animaux constituent un danger physique pour l'homme. Sans oublier les animaux de l'ombre comme les araignées et les serpents, ou encore ces guêpes, fourmis, aoûtats, autant de nuisances insupportables. Mais également tous ces parasites ou leurs vecteurs, d'autant plus redoutables qu'ils sont peu visibles et qu'on ne les repère pas facilement. Et aussi tous les micro-organismes responsables de terribles épidémies chez l'homme et ses animaux domestiques! Sans oublier ces 
A paraître dans la revue : Recherches en Didactique des Sciences et des Technologies.

nombreux ravageurs des cultures qui ont parfois anéanti les récoltes et mené des pays entiers à la famine et la misère. La liste est loin d'être close ! Elle est même bien longue étant donné la grande diversité des parasites et des micro-organismes pathogènes.

Face à ces agressions l'homme a réagi par la peur, la fureur, la haine contre les bêtes ennemies. Il a d'abord cherché à les repousser, à les effrayer par l'usage du feu, en lançant des pierres, ou en apprivoisant d'autres animaux comme le chien. [...] L'homme n'a pas hésité à utiliser les poisons et les pièges pour arriver à ses fins. Il a développé des industries puissantes pour fabriquer du DDT et des milliers de pesticides, ou pour fabriquer des médicaments permettant de lutter contre les maladies. Au point de mettre en péril sa propre existence par les pollutions ainsi engendrées.

[...]

[O]n doit se rappeler la détresse des contemporains confrontés à la famine suite à des invasions d'insectes et de rongeurs dévastant leurs cultures, à des époques où l'on était complètement désarmé face à de tels phénomènes.

[...]

Bref, un fossé existe bel et bien entre la vision d'une nature idyllique et la réalité quotidienne des hommes confrontés aux assauts de la diversité biologique. II ne faut pas se voiler la face : leur première préoccupation est de se débarrasser de toutes ces espèces qui menacent leur santé, leurs récoltes, leurs biens.

\section{Annexe 4 : Exemple d'un test complété en classe de SVT :}

Que penses-tu de cette affirmation ?

"II ne faut pas changer les activités humaines qui permettent le développement économique et social uniquement parce qu'elles pourraient faire disparaître des espèces animales ou végétales. "

$\begin{array}{cccc}\square & \square & \square & \square \\ \begin{array}{c}\text { Tout à fait } \\ \text { d'accord }\end{array} & \begin{array}{l}\text { Neutre / Je } \\ \text { d'accord }\end{array} & \begin{array}{c}\text { Plutôt pas } \\ \text { d'accord }\end{array} & \begin{array}{c}\text { Pas du tout } \\ \text { d'accord }\end{array}\end{array}$

\section{Pourquoi ?}

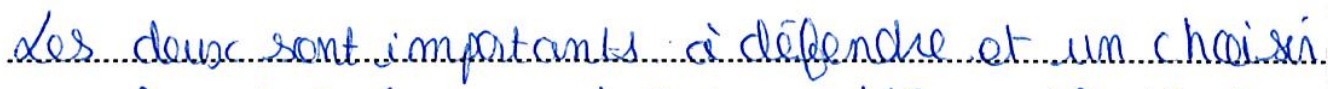

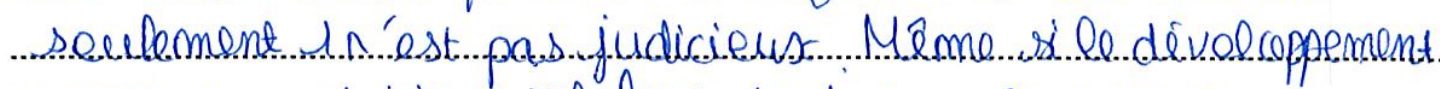

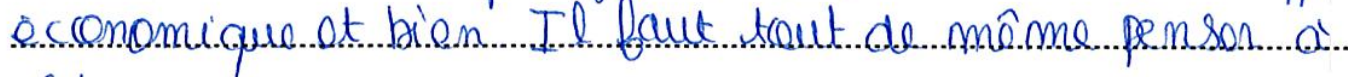
l...'onlujisonnomont. 